\title{
Evolution of Songbirds (Passeriformes) and their Presence in the Neogene and the Quaternary in the Carpathian Basin
}

\author{
Jenő (E.) KESSLER
}

Received: March 09, 2020 - August 31, 2020 - September 01, 2020

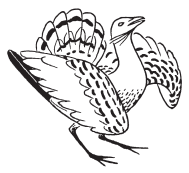

Kessler, J. (E). 2020. Evolution of songbirds (Passeriformes) and their presence in the Neogene and the Quaternary in the Carpathian Basin. - Ornis Hungarica 28(2): 158-203. DOI: 10.2478/ orhu-2020-0024

\begin{abstract}
Songbirds are the largest order of birds with 6456 species, making up more than half of every known bird species. The location and time of their emergence, as well as the method of their spreading, is debated. They are present in the Carpathian Basin from the beginning of the Neogene, with an increasing number of types and species. Due to their diverse ways of life and diets, their presence mirrors the environmental conditions of the given geological periods quite accurately.
\end{abstract}

Keywords: Perching birds, Tertiary, Quaternary, Paleogene, Neogene, avian fauna

Összefoglalás A verébalakúak a madarak osztályának legnépesebb rendje 6456 fajjal, ami több mint a felét képviseli az összes ismert madárfajnak. Megjelenésük helye, ideje és elterjedésük módja vitatott. A Kárpát-medencében a Neogén elejétől vannak jelen, növekvő típus- és fajszámban. Változatos életmódjuk és táplálkozásuk következtében jelenlétük jól tükrözi az adott földtörténeti időszak környezeti viszonyait.

Kulcsszavak: énekesmadarak, Harmadidőszak, Negyedidőszak, Paleogén, Neogén, madárfauna

Department of Paleontology, Eötvös Loránd University, 1117 Budapest, Pázmány Péter sétány 1/c, Hungary, e-mail: kessler_jeno@yahoo.com

\section{Introduction}

About half of the extant avian species consists of songbirds, which can be found all around the world, apart from Antarctica, with a large number of species. They supposedly formed in the area of the ancient continent of Gondwana (Australia and surroundig archipelago) but their fossil remains in the Palearctic and Nearctic are only known from the Paleogene-Neogene boundary in small numbers. Extantly, their research received a boost, and so did the number of identified and described extant and extinct taxa (Kessler 2013a, 2013b, 2015).

According to the current status of science, songbirds spread from Australia and its environs to the whole world during the Oligocene. The earliest known Palaearctic remains come from the Upper Oligocene (MP 30) from sites at Coderet and Gannat (Allier) in France (Mourer-Chauviré et al. 1989). Typically, these finds already bear the osteomorphological signs indicating passerines (Nagy 2020). Thereafter, fossils from Europe, Asia, and also from North and South America are only known from the Lower Miocene. In South America, passerine remains have been described from the Lower Miocene in Patagonia (Noriega \& Chiappe 1993). This could suggest that the spread of the passerines ended around this time. 
Fossils from the northern hemisphere from before the Miocene probably do not belong to songbirds, but rather to Coraciiformes species that were dominantly present in the Paleogene (Olson 1985). The first songbird fossils were described by Milne-Edwards (1869-71) from the Lower Miocene of France (named Motacilla humata, M. major, Lanius miocaenicus). Brodkorb (1978) noted finds from the Lower Miocene of Florida as well, while Howard (1957) described the species Palaeoscines turdirostris from the Late Miocene of California. Numerous finds had been noted from Europe, but only defined to the family or genus level (Ballman in 1972 noted Alauda and Sitta species from the Early Miocene of France in 1972, while Steadman noted Emberizinae species from the Miocene of the USA in 1982).

Despite having prior finds rich in songbird remains, their definitions only go to the family or genus level at most, with only a few exceptions. One of the main reasons for this is the highly significant phenotypical homogenity, resulting in the morphological or size differences having been handled by experts as unique characteristics. The other reason is the sentiment that species do not modify or shift into new species even after many thousands/tens of thousands of generations. This approach also disregards the fact that Linnaean taxonomy distinguishes between related species primarily based on outer morphological/phenotypical characteristics (the form of the beak, colors of feathers, dimensions, etc.), but skeletal characteristics do not play a part in the diagnostics of species. These differences can only be shown via comparative skeletal examinations; these had not been undertaken until extantly, and even those that have only compared particular skeletal parts (in the articles of Fürbringer, Lambrecht, Jánossy and others, also cited in this work).

The osteological characteristics, however, only hint at movement and physiological roles, apart from the beaks that hint at their diets. As opposed to this, the feathers that have a main role in species diagnostics are significant in camouflage and mating. Typically species with open nests either the color of both males or females is gray-brown, or the color of females brooding at daytime is significantly less garish than that of males. In the case of songbird species brooding in closed nests there is no significant difference between the colors of the two genders, although the color of males might be more vibrant even there. The change in species originating from environmental change mostly has an effect on the feathers during reproduction and camouflage to avoid predators (primarily in times of brooding and raising their young), but this cannot be shown in the case of remains significant to paleontology. Moreover, the changes in skeletal parts (mostly the proportion and size of limbs) can only show differences in movement, which is not necessarily typical when a new species is formed. Non-adaptive new colors, voice or mating dances can be detrimental when searching for a mate (for example, albino specimens have no osteological differences, but the lack of species-specific colors has a significant negative effect in mating).

Paleontological finds do not show the characteristics on which the Linnaean taxonomy is based, and due to their age, molecular genetic classification is also impossible. Thus, the specialists can only define the material and create new genera, species, subspecies based on available osteological characteristics/differences/similarities, or accept the opinion of many that during the last millions of years no shift in species took place regarding these taxonomical types. This latter view not only opposes the theory of evolution, but also disregards the 
fact that members of the order of songbirds are sexually mature by their first year, forming one or several new generations every year.

It is hard to imagine that during millions of generations, no changes would take place that would form new species with different characteristics to their distant ancestors. This is also refuted by the current diversification of finches on the Galapagos Islands.

In this work, the author examined and identified several thousand skeletal parts of songbirds in the Carpathian Basin from the Lower, Middle and Upper Miocene, the Pliocene and Lower Pleistocene, describing more than 120 new species, mostly from sites located in Hungary. Most of the examined bones come from earlier collections, but they were only identified to the family/genus level at best.

It is a welcome news that possibly due to the newly described species in 2012 and 2015 , as well as the osteological guide to the genus level published in 2015, the classification of the remains of the order down to the species level and their publication has seen a steady rise worldwide, resulting in more and more studies of this nature.

Abbreviations: MN 1-5 (23,5-16,5 MY) - Lower Miocene; MN 6-8 (16,5-11,5 MY) Middle Miocne; MN 9-13 (11,5-5,3 MY) - Upper Miocene; MN 14-15 (5,3-3,2 MY) Lower Pliocene; MN 16-17 (3,2-1,8 MY) - Upper Pliocene; Q1-Q2 (1,8 MY-500.000Y) - Lower Pleistocene; Q3 (Q3/I-Q3/II) (500.000-120.000Y) - Middle Pleistocene; Q4/I (120.000-15.000Y) - Upper Pleistocene; Q4/II (15.000Y) - Holocene; $\uparrow$ - extinct/fossil species - subspecies.

In the geochronological sense we use the early, middle and late prefixes when dividing the periods into ages, and in the chronostratigraphic sense we use the lower, middle and upper prefixes when dividing the systems into series.

\section{Systematics}

Ord. Passeriformes Linnaeus, 1758

Fam. Alaudidae (Vigors, 1825)

- Melanocorypha Boie, 1826

- Melanocorypha $\uparrow$ minor Kessler, 2013

Type locality and age: Beremend 26, Pliocene (MN 15) (Hungary) (Kessler 2013a, 2013b). It corresponds to the extant genus, but dimensions are smaller.

- Melanocorypha calandra (Linnaeus, 1766)

Q1: Betfia 9 (Romania) (Gál 2002).

From sites in Europe outside the Carpathian Basin Q3: France, Russia, Spain; Q4: France, Italy, Moldova, Poland, Spain, Ukraine (Tyrberg 1998).

- Melanocorypha sp. indet.

Q1: Beremend 17 (Hungary) (Jánossy 1992, 1996).

From sites in Europe outside the Carpathian Basin:

- Melanocorypha bimaculata (Ménétries, 1832)

Q3: Azerbaijan (Tyrberg 1998). 


\section{- Melanocorypha maxima Blyth, 1867}

Q4: Italy (Tyrberg 1998).

\section{- Melanocorypha leucoptera (Pallas, 1811)}

Q4: Germany (Tyrberg 1998).

- Melanocrorypha yeltoniensis (Forster, 1767)

Q3: Russia; Q4: Ukraine (Tyrberg 1998).

The genus was reported from Bulgaria: Melanocorypha serdicensis Boev, 2012 (Upper Miocene, Hrabarsko) and Melanocorypha donchevi Boev, 2012 (Upper Pliocene, Varshets) (Boev 2012). One fossil species of larks have been described from the Pleistocene deposits in Israel: Melanocorypha gracilis Tchernov, 1968 (Tyrberg 1998).

- Galerida Boie, 1828

- Galerida † cserhatensis Kessler et Hír, 2012

Type locality and age: Litke 2, Lower Miocene (MN 5) (Hungary) (Kessler \& Hír 2012); It largely corresponds to the extant Galerida cristata, with small morphological differences.

- Galerida † pannonica Kessler, 2013

Type locality and age: Csarnóta 2, Pliocene (MN 15) (Hungary) (Kessler 2013a, 2013b).

Other locality and age: Beremend 26, Pliocene (MN 15) (Hungary) (Kessler 2013a, 2013b).

It corresponds in characteristics and sizes with extant species of the genus.

\section{- Galerida cristata (Linnaeus, 1758)}

Q1: Betfia 2, 9 (Romania) (Kormos 1913, Čapek 1917, Lambrecht 1933, Kessler 1975, Jánossy 1979, Gál 2002); Q2: Somssich-hegy 2 (Hungary) (Jánossy 1981, 1983, 1986); Q4/I: Hámor-Puskaporos Niche (Lambrecht 1912, 1916, 1933, Jánossy 1979, 1986); TatabányaKálváriahegy 4. Cave (Gál 2005a, 2005b) (all in Hungary); Q4/II: Hosszúhegyi Cave (Hungary) (Jánossy 1979); Peterd-Tordai Defile - Magyar Cave (Petrești, Cheile Turzii-Peștera Ungurească) (Romania) (Kessler \& Gál 1998, Gál 2005a). From sites in Europe outside the Carpathian Basin Q3: France, Germany, Spain; Q4: Bosnia-Herczegovina, Czech Republic, France, Germany, Moldova, Poland, Russia, Spain, Ukraine, United Kingdom.

- Galerida sp. indet.

Q1: Beremend 17 (Hungary) (Jánossy 1992, 1996).

From sites in Europe outside the Carpathian Basin:

\section{- Galerida theklae (Brehm, 1858)}

Q3: Spain; Q4: Spain (Tyrberg 1998).

The genus was reported outside the Carpathian Basin in Bulgaria from Varshets (Upper Pliocene, MN 17) as Galerida bulgarica Boev, 2012 (Boev 2012). It is also known with extant species only from Middle Pleistocene in European fossil localities (Tyrberg 1998).

- †Praealauda Kessler et Hír, 2012

- †Praealauda hevesensis Kessler et Hír, 2012

Type locality and age: Felsőtárkány, Middle Miocene (MN 7-8) (Hungary) (Kessler \& Hír 2012).

A new genus and species of the Alaudidae family. Originally, was marked as Turdus sp. indet. (Hír et al. 2001). 


\section{- Alauda Linnaeus, 1758}

\section{- Alauda † tivadari Kessler, 2013}

Type locality and age: Polgárdi 4, Late Miocene (MN 13) (Hungary) (Kessler 2013a, 2013b). The coracoid is somewhat smaller than in extant species, while the distal fragment of the tibiotarsus is equal to it in the size. The extinct species from Felsőtárkány (MN 7-8): Praealauda hevesensis Kessler et Hír, 2012 differs in its age, sizes and morphological characteristics (Kessler \& Hír 2012).

\section{- Alauda arvensis Linnaeus, 1758}

Q1: Beremend 16 (Hungary) (Jánossy 1992, 1996); Betfia 2, 9 (Romania) (Kormos 1913, Čapek 1917, Lambrecht 1933, Kessler 1975, Jánossy 1979, Gál 2002); Q3/I: Hundsheim (Austria) (Lambrecht 1933, Jánossy 1974a, 1979); Q4/I: Hámor-Puskaporos Niche (Hungary) (Lambrecht 1912, 1916, 1933, Jánossy 1979, 1986); Detrekőszentmiklós-Pálffy Cave (Dzeráva Skála-Plavecky Mikulas) (Slovakia) (Lambrecht 1913, 1933);

Q4/II: Ecsegfalva (Hungary) (Pike-Tay et al. 2004, Gál 2007); Gyulafehérvár (Alba Iulia) (Gál 2005a, 2005b); Szegyestel-Drăcoaia Cave (Sighiştel, Peştera Drăcoaia) (Kessler 1982) (all in Romania). From sites in Europe outside the Carpathian Basin Q1-2: France, Ukraine; Q3: Austria, Czech Republik, France, Italy, Russia, Spain; Q4: Austria, Bulgaria, Czech Republic, Italy, Poland, Russia, Spain, Switzerland, Ukraine, United Kingdom (Tyrberg 1998).

In the Carpathian Basin, the extant genus and species are also known from the Early Pleistocene in Hungary (Beremend 16), Romania (Betfia 9) (Jánossy 1992, Gál 2002). The genus was reported extantly outside the Carpathian Basin in Bulgaria (Upper Pliocene, MN 17, Varshets) as Alauda xerarvensis Boev, 2012 (Boev 1996, 2012) and is also known with extant species from the Early Pleistocene from Valerots (France) and Stránská skála (Czech Republic) (Tyrberg 1998). Alauda gypsorum Portis, 1887 and Alauda major Portis, 1887 (Portis 1887) from the Late Miocene (MN 13) of Seniglia and Gabbro (Italy) were reported in slab as fossil species, but Mlíkovsky (2002) put them into „Family incertae sedis".

\section{- Lullula Kaup, 1829}

\section{- Lullula † neogradensis Kessler et Hír, 2012}

Type locality and age: Mátraszőlős 1, Middle Miocene (MN 7-8) (Hungary) (Kessler \& Hír 2012).

An extinct Lullula species previously was identified as Pyrrhula sp. (Gál et al. 1999).

\section{- Lullula †minor Kessler, 2013}

Type locality and age: Polgárdi 4, 5; Upper Miocene (MN 13) (Hungary) (Kessler 2013a, 2013b).

The fossil species differs from extant with its smaller sizes and in some morphological characteristic. The fossil species Lullula neogradensis Kessler et Hír, 2012 from Mátraszőlös was described based on other bones and its age is much older (Kessler \& Hír 2012).

\section{- Lullula † parva Kessler, 2013}

Type locality and age: Csarnóta 2, Pliocene (MN 15) (Hungary) (Kessler 2013a, 2013b). Other locality and age: Beremend 26, Pliocene (MN 15) (Hungary) (Kessler 2013a, 2013b). 
It corresponds in characteristics to the extant genus. The fossil species Lullula neogradensis Kessler et Hír, 2012 from Mátraszőlős 1 and Lullula † minor from Polgárdi differ in its age and sizes to Csarnótian and Beremendian specimens.

\section{- Lullula $\dagger$ minuscula n. sp.}

Type locality and age: Beremend 26, Pliocene (MN 15) (Hungary) (Kessler 2013a, 2013b). In size corresponds with Lullula minor Kessler, 2013 from Polgárdi but is younger in age. It is different in size and in characteristics to Lullula parva.

\section{- Lullula arborea (Linnaeus, 1758)}

Q1: Betfia 2, 9 (Romania) (Kormos 1913, Čapek 1917, Lambrecht 1933, Kessler 1975, Jánossy 1979, Gál 2002). From sites in Europe outside the Carpathian Basin Q1-2: Spain; Q3: France, Italy, Spain; Q4: Belgium, Bulgaria, Czech Republic, France, Italy, Poland, Russia, Spain, Ukraine, United Kingdom (Tyrberg 1998).

The genus was reported outside the Carpathian Basin in Bulgaria from the Late Miocene Chrabarsko as Lullula sp. (Boev 2000), and from the Late Pliocene - Early Pleistocene as Lullula slivnicensis Boev, 2012 (Slivnica, MN 17) and L. balcanica Boev, 2012 (Varshets, MN 18) based on other skeletal types (Boev 1996, 2012). The extant species Lullula arborea was reported from the Late Pliocene and the Early Pleistocene (MN 18) in Mallorca (Spain) (Sondaar et al. 1995), but probably they are also fossil species.

\section{- Calandrella Kaup, 1829}

- Calandrella $\uparrow$ gali Kessler, 2013

Type locality and age: Polgárdi 4, 5; Upper Miocene (MN 13) (Hungary) (Kessler 2013a, 2013b). It corresponds in its characteristics to extant species but its size is somewhat different. The genus was reported only from sites in Europe outside the Carpathian Basin:

- Calandrella cinerea (J. F. Gmelin, 1789)

Q1-2: Ukraine; Q4: Spain, Ukraine (Tyrberg 1998).

- Calandrella brachydactyla (Leisler, 1814)

Q3: France, Italy; Q4: France, Greece (Tyrberg 1998).

- Calandrella rufescens (Viellot, 1820)

Q3: Azerbaijan (Tyrberg 1998).

\section{- Eremophila Boie, 1828}

- Eremophila alpestris (Linnaeus, 1758)

Q4/I: Gencsapáti (Hungary) (Jánossy 1979); Q4/II: Grosse Offenbergerhöhle (Austria), (Bocheński \& Tomek 1994). From sites in Europe outside the Carpathian Basin Q1-2: France; Q3: France, Germany, Russia; Q4: Belgium, Czech Republic, Germany, Italy, Poland, Russia, Switzerland, Ukraine, United Kingdom (Tyrberg 1998).

The genus was reported from Bulgaria: Eremarida xerophila Boev, 2012 (Upper Miocene, Hrabarsko); Eremophila prealpestris Boev, 2012 (Upper Pliocene, Varshets) (Boev 1996, 2012). The extant species Eremophila alpestris (Linnaeus, 1758) was described from the Late Pliocene of Mas Ramboult (France) (Mourer-Chauviré 1975). Finally, Zelenkov (2011) reported the Eremophila aff. E. alpestris in the Late Pliocene (MN 16) of Beregovaya (Bichursky District, Republic of Buryatia, Russia). 


\section{Fam. Hirundinidae Vigors, 1825}

\section{- Hirundo Linnaeus, 1758}

\section{- Hirundo $\uparrow$ gracilis Kesler, 2013}

Type locality and age: Polgárdi 4, 5; Upper Miocene (MN 13) (Hungary) (Kessler 2013a, 2013b). More slender than the extant species.

\section{- Hirundo † major Kessler, 2013}

Type locality and age: Csarnóta 2, Pliocene (MN 15) (Hungary) (Kessler 2013a, 2013b). It corresponds in characteristics with extant genus, but its dimensions are larger. This material was reported as Hirundo sp. from Csarnóta 2 by Jánossy $(1972,1979)$. The fossil species Hirundo † gracilis Kessler, 2012 from Polgárdi is smaller than the Csarnótian specimen.

\section{- Hirundo rustica Linnaeus, 1758}

Q1: Németóvár 4B (Austria) (Jánossy 1981, Döppes \& Rabeder 1997, Mlikovskỳ 1998); Osztramos 8 (Hungary) (Jánossy \& Kordos 1976); Betfia 2, 9 (Romania) (Kormos 1913, Čapek 1917, Lambrecht 1933, Kessler 1975, Jánossy 1979, Gál 2002); Q2: Somssich-hegy 2 (Hungary) (Jánossy 1981, 1982, 1983, 1986); Méhész (Vcelare) (Slovakia) (Horáček 1985, Mlikovskỳ 2002); Q3/I: Hundsheim (Austria) (Lambrecht 1933, Jánossy 1974a, 1979, Mlikovskỳ 1998, 2002); Vindija (Croatia) (M. Malez 1961, V. Malez 1973, 1988); Betfia 7/4 (Romania) (Kessler 1975, Jánossy 1979, Gál 2002); Q4/I: Budapest-Remetehegyi Niche (Kormos 1914, Lambrecht 1933, Jánossy 1979, 1986), Pilisszántó I. Niche (Lambrecht 1915, 1933, Jánossy 1979, 1986), Szilvásvárad-Istállóskői Cave (Lambrecht 1912, 1933, Jánossy 1952, 1955, 1979, 1986), Tatabánya-Kálváriahegy 4. Cave (Gál 2005a, 2005b) (all in Hungary); Ohábaponor-Bordu Mare Cave (Ohaba, Peștera Bordu Mare) (Kessler 1985, Jurcsák \& Kessler 1988, Gál 2002, 2003), Szegyestel-Măgura Cave (Sighiștel, Peștera Măgura) (Kessler 1982, 1985, Gál 2002) (all in Romania); Q4/II: Grosse Offenbergerhöhle (Austria) (Bocheński \& Tomek 1994); Hosszúhegyi Cave (Hungary) (Jánossy 1979); Herkulesfürdő-Rablók Cave (Băile Herculane, Peştera Hoţilor) (Kessler 1981, Gál 2002), Révi Caves (Peşterile din Vadu Crişului) (Kessler 1982), Szegyestel-Drăcoaia Cave (Sighiştel, Peştera Drăcoaia) (Kessler 1982) (all in Romania). From sites in Europe outside the Carpathian Basin Q3: Czech Republic, France, Germany, Italy, Russia, Spain, United Kingdom; Q4: Austria, Belgium, Croatia, Czech Republic, France, Germany, Greece, Ireland, Italy, Poland, Russia, Spain, Ukraine, United Kingdom (Tyrberg 1998).

- Hirundo sp. foss. indet.

MN 15: Beremend 26 (Hungary) (Kessler 2010); Ivánháza I (Ivanovce I) (Slovakia) (Mlíkovskỳ 2002); Q1: Beremend 16 (Hungary) (Jánossy 1992, 1996).

\section{- Hirundo sp. indet.}

Q2: Nagyharsányhegy 1-4 (Hungary) (Lambrecht 1916, 1933, Jánossy 1978, 1979, 1980); Q3/II: Cserépfalu-Hórvölgyi Cave (Hungary) (Jánossy 1979). From sites in Europe outside the Carpathian Basin Q3: Italy, Malta; Q4: France, Germany, Italy, Poland, Russia, Spain, Switzerland, Ukraine, United Kingdom (Tyrberg 1998).

\section{- Hirundo rupestris Scopoli, 1789}

Q4/I: Cserépfalu-Subalyuk Cave (Hungary) (Jánossy 1979). 
From sites in Europe outside the Carpathian Basin Q3: France, Spain; Q4: Belgium, Bulgaria, France, Georgia, Germany, Italy, Poland, Spain, Switzerland (Tyrberg 1998).

- Hirundo daurica Linnaeus, 1771

From sites in Europe outside the Carpathian Basin Q3: France; Q4: Croatia, France, Germany, Greece, Italy (Tyrberg 1998).

- Delichon Moore, 1854

- Delichon $\uparrow$ polgardiensis Kessler, 2013

Type locality and age: Polgárdi 5, Upper Miocene (MN 13) (Hungary) (Kessler 2013a, 2013b). It corresponds in its characteristics to extant species but differs in its sizes.

\section{- Delichon $\uparrow$ pusillus Kessler, 2013}

Type locality and age: Csarnóta 2, Pliocene (MN 15) (Hungary) (Kessler 2013a, 2013b). It is smaller as the extant species but mostly corresponds to it in the characteristics.

\section{- Delichon $\dagger$ major Kessler, 2013}

Type locality and age: Beremend 26, Pliocene (MN 15) (Hungary) (Kessler 2013a, 2013b). It corresponds in characteristics with extant species, but has larger sizes.

- Delichon urbica (Linnaeus, 1758)

Q1: Betfia 2, 9 (Romania) (Kormos 1913, Čapek 1917, Lambrecht 1933, Kessler 1975, Jánossy 1979, Gál 2002); Q4/I: Tatabánya-Kálváriahegy 4. Cave (Hungary) (Gál 2005a, 2005b); Q4/II: Grosse Offenbergerhöhle (Austria) (Bocheński \& Tomek 1994); Révi Caves (Peşterile din Vadu Crișului) (Romania) (Kessler 1982). From sites in Europe outside the Carpathian Basin Q1-2: Czech Republic (Stránská skála, Mlíkovskỳ 1995) and Spain (Quibas) (Montoya et al. 1999); Q3: Russia, Ukraine; Q4: Croatia, Czech Republic, France, Greece, Ireland, Italy, Russia, Ukraine, United Kingdom (Tyrberg 1998).

\section{- Riparia Forster, 1817}

\section{- Riparia $\uparrow$ minor Kessler, 2013}

Type locality and age: Polgárdi 4, Upper Miocene (MN 13) (Hungary) (Kessler 2013a, 2013b). Differs from extant species in some morphological characteristics and in its smaller sizes.

\section{- Riparia riparia (Linnaeus, 1758)}

Q1: Betfia 2, 9 (Romania) (Kormos 1913, Čapek 1917, Lambrecht 1933, Kessler 1975, Jánossy 1979, Gál 2002). From sites in Europe outside the Carpathian Basin, the species is known from the Early Pleistocene (Q1) from Czech Republic (Stránská skála) (Mlikovskỳ 1995); Q3: France; Q4: France, Italy (Tyrberg 1998).

\section{Fam. Paridae Boie, 1826}

- Aegithalos Hermann, 1804

- Aegithalos $\uparrow$ gaspariki Kessler, 2013

Type locality and age: Polgárdi 4, 5; Upper Miocene (MN 13) (Hungary) (Kessler 2013a, 2013b). It corresponds more in characteristics and sizes to extant species of the genus.

\section{- Aegithalos $\dagger$ congruis Kessler, 2013}

Type locality and age: Csarnóta 2, Pliocene (MN 15) (Hungary) (Kessler 2013a, 2013b). It mostly corresponds with extant species in the characteristics and sizes. 


\section{- Aegithalos caudatus (Linnaeus, 1758)}

Q1: Betfia 2 (Romania) (Kormos 1913, Čapek 1917);

The genus is known in fossil material with extant species in Q1-2: from S'Onix-Mallorca - Spain (Sondaar et al. 1995); Q3: France; Q4: Italy, Poland, Ukraine (Tyrberg 1998).

- Parus Linnaeus, 1758

- Parus $\uparrow$ medius Kessler, 2013

Type locality and age: Beremend 26, Pliocene (MN 15) (Hungary) (Kessler 2013a, 2013b). It is among the medium-sized tits.

- Parus $\uparrow$ robustus Kessler, 2013

Type locality and age: Csarnóta 2, Pliocene (MN 15) (Hungary) (Kessler 2013a, 2013b). It is fossil species with larger dimensions than extant Parus major.

\section{- Parus † parvulus Kessler, 2013}

Type locality and age: Csarnóta 2, Pliocene (MN 15) (Hungary) (Kessler 2013a, 2013b). It is small tit species.

\section{- Parus coeruleus Linnaeus, 1758}

Q1: Betfia 9 (Romania) (Gál 2002); Q4/I: Mixnitz - Drachenhöhle (Austria) (Lambrecht 1933). From sites in Europe outside the Carpathian Basin Q3: Czech Republic; Q4: Germany (Tyrberg 1998).

\section{- Parus major Linnaeus, 1758}

Q1: Betfia 2, 9 (Romania) (Kormos 1913, Čapek 1917, Lambrecht 1933, Kessler 1975, Jánossy 1979, Gál 2002); Q2: Kiskóh-Medve Cave (Chiscäu, Peștera Urșilor) (Romania) (Kessler 1982, Jurcsák \& Kessler 1988, Gál 2002); Q3/I: Hundsheim (Austria) (Lambrecht 1933, Jánossy 1974a, 1979); Vindija (Croatia) (M. Malez 1961, V. Malez 1973, 1988); Q4/I: Merkenstein (Austria) (Wettstein \& Mülhofer 1938); Velika Pecina (Croatia) (V. Malez 1975, 1984, 1988); Körösmart (Rîpa) (Romania) (Jánossy in Hamar \& Csák 1969, Kessler 1974a, Gál 2002); Q4/II: Hosszúhegyi Cave (Hungary) (Jánossy 1979). From sites in Europe outside the Carpathian Basin Q1-2: Spain, Ukraine; Q4: Austria, Bosnia-Herczegovina, Croatia, Czech Republic, France, Germany, Spain, Ukraine, United Kingdom (Tyrberg 1998).

\section{- Parus lugubris Temminck, 1820}

Q1: Betfia 2 (Romania) (Kormos 1913, Čapek 1917).

\section{- Parus ater Linnaeus, 1758}

Q3: Tarkő 1 (Hungary) (Jánossy 1979). From sites in Europe outside the Carpathian Basin Q1-2: Spain, Q4: Czech Republic, France, Ukraine (Tyrberg 1998).

\section{- Parus palustris Linnaeus, 1758}

Q3: Hundsheim (Austria) (Lambrecht 1933, Jánossy 1974a, 1979). From sites in Europe outside the Carpathian Basin Q3: Germany; Q4: Austria, Croatia (Tyrberg 1998).

\section{- Parus montanus Conrad, 1827}

Q4/I: Velika Pecina (Croatia) (V. Malez 1975, 1984, 1988). From sites in Europe outside the Carpathian Basin Q4: Austria, France (Tyrberg 1998).

\section{- Parus cristatus Linnaeus, 1758}

Only from sites in Europe outside the Carpathian Basin Q1-2: Spain; Q3: France, Spain; Q4: France, Poland, Ukraine (Tyrberg 1998). 


\section{- Parus sp.}

Q3/II: Uppony I/1 (Hungary) (Jánossy 1979); Q4/I: Érd (Hungary) (Jánossy 1979). From sites in Europe outside the Carpathian Basin Q4: Ukraine (Tyrberg 1998).

- Panurus Koch, 1816

- Panurus biarmicus (Linnaeus, 1758)

From sites in Europe outside the Carpathian Basin Q4: Germany (Tyrberg 1998). The family is known outside the Carpathian Basin only from the Late Pliocene from Varsets (MN 17, Bulgaria) as Parus sp. (Boev 2000).

\section{Fam. Sittidae Bonaparte, 1831}

- Sitta Linnaeus, 1758

- Sitta $\dagger$ gracilis Kessler, 2013

Type locality and age: Polgárdi 4, Upper Miocene (MN 13) (Hungary) (Kessler 2013a, 2013b). Its size is smaller than in extant species.

\section{- Sitta $\uparrow$ pusilla Kessler, 2013}

Type locality and age: Csarnóta 2, Pliocene (MN 15) (Hungary) (Kessler 2013a, 2013b). The dimensions of fossil species are smaller than those of extant species. The Sitta gracilis from Polgárdi was described also on the basis of carpometacarpus. It is larger than the Csarnótian specimen and differs from it in shape of the processus extensorius; that of the Polgárdi specimen is shorter than in the Csarnótian remains. The processus alularis is more pointed. The characteristics correspond in general to extant species. Jánossy (1995) reported these bones as Sitta sp.

\section{- Sitta † villanyensis Kessler, 2013}

Type locality and age: Beremend 26, Pliocene (MN 15) (Hungary) (Kessler 2013a, 2013b). It corresponds in characteristics and size with extant species and differs in these to extinct species from Polgárdi and Csarnóta, which are much smaller. Previously was reported as Sitta sp. foss. indet. (Kessler 2010).

\section{- Sitta europaea Linnaeus, 1758}

Q1: Németóvár 4B (Austria) (Jánossy 1981); Betfia 9 (Romania) (Gál 2002); Q2: Somssich-hegy 2 (Hungary) (Jánossy 1981, 1982, 1983, 1986); Q4/I: Velika Pecina (Croatia) (V. Malez 1975, 1984, 1988); Q4/II: Bodajk-Rigólyuk (Hungary) (Kordos 1984); Szkerisoara-Coiba Mare Cave (Scărişoara, Peştera Coiba Mare) (Romania) (Kessler 1985). From sites in Europe outside the Carpathian Basin Q3: Czech Republic, Ukraine; Q4: Austria, Bosnia-Herczegovina, Bulgaria, Croatia, Czech Republic, France, Poland, Ukraine (Tyrberg 1998).

- Sittidae gen et sp. foss. indet.

MN 6: Köalja 2 (Subpiatra 2) (Romania) (Kessler \& Venczel 2009).

The genus is known outside the Carpathian Basin only from the Early Pliocene (MN 16) from Rebielice Królowskie I. (Poland) as Sitta sp. (also with smaller sizes) (Jánossy 1974b) and from the Late Pliocene from Varsets (MN 17, Bulgaria) (Boev 1996, 2000). The fossil species Sitta senogalliensis Portis, 1887 from Senigallia (Upper Miocene, MN 13, Italy) was put by Mlíkovsky (2002) into "Family incertae sedis". 


\section{Fam. Certhiidae Vigors, 1825}

\section{- Certhia Linnaeus, 1758}

- Certhia † janossyi Kessler et Hír, 2012

Type locality and age: Rudabánya, Upper Miocene (MN 9) (Hungary) (Kessler \& Hír 2012). It corresponds with extant species in the characteristics and sizes.

\section{- Certhia † immensa Kessler, 2012}

Type locality and age: Csarnóta 2, Pliocene (MN 15) (Hungary) (Kessler 2013a, 2013b). The sizes are larger than in extant species. The fossil material was reported previously as Certhia sp. (Kessler 2010).

\section{- Certhia familiaris Linnaeus, 1758}

Q1: Betfia 2 (Romania) (Kormos 1913, Čapek 1917, Lambrecht 1933, Kessler 1975, Jánossy 1979, Gál 2002). From sites in Europe outside the Carpathian Basin as Certhia sp. Q4: France, Germany (Tyrberg 1998).

- Certhiidae gen. et sp. foss. indet.

MN 6: Kőalja 2 (Subpiatra 2) (Romania) (Kessler \& Venczel 2009).

The family and genus was reported with fossil species only from the Carpathian Basin.

\section{Fam. Tichodromidae Swainson, 1827}

\section{- Tichodroma Illiger, 1811}

- Tichodroma $\uparrow$ capeki Kessler, 2013

Type locality and age: Polgárdi 4, Upper Miocene (MN 13) (Hungary) (Kessler 2013a, 2013 b). Its characteristics correspond to extant species. The family and genus were reported as fossil and extant species only from the Carpathian Basin and from Q4: Italy, Poland (Tyrberg 1998).

\section{Fam. Muscicapidae Vigors, 1825}

- Muscicapa Linnaeus, 1766

- Muscicapa † leganyii Kessler et Hír, 2012

Type locality and age: Felsőtárkány, Middle Miocene (MN 7-8) (Jánossy 1979) (Kessler \& Hír 2012). Other locality and age: Felnémet 2/3, Middle Miocene (MN 7-8) (Hungary) (Kessler \& Hír 2012).

It corresponds with extant species sizes.

\section{- Muscicapa $\dagger$ miklosi Kessler, 2013}

Type locality and age: Polgárdi 4, Upper Miocene (MN 13) (Hungary) (Kessler 2013a, 2013b). It corresponds in characteristics and sizes to extant species of the genus.

- Muscicapa † petényii Kessler, 2013

Type locality and age: Beremend 26, Pliocene (MN 15) (Hungary) (Kessler 2013a, 2013b). It corresponds in characteristics and in dimensions to extant species of Muscicapa (Ficedula) genus.

- Muscicapa aff. striata (Pallas, 1764)

Q1: Betfia 9 (Romania) (Gál 2002); Q3/I: Hundsheim (Austria) (Lambrecht 1933, Jánossy 1974a, 1979); Q4/II: Bodajk-Rigó Niche (Kordos 1984), Hosszúhegyi Cave (Jánossy 1979) (all in Hungary). 
From sites in Europe outside the Carpathian Basin Q4: Belgium, Greece (Tyrberg 1998).

- Ficedulla albicollis (Temminck, 1815)

From sites in Europe outside the Carpathian Basin Q3: France, Germany; Q4: France, Ukraine (Tyrberg 1998).

- Ficedula hypoleuca (Pallas, 1764)

From sites in Europe outside the Carpathian Basin Q3: France; Q4: France (Tyrberg 1998). The genus is known in Late Pliocene - Early Pleistocene boundary (MN 17-18) from S'Onix - Mallorca (Spain) as Muscicapa striata (Pallas, 1764) (Sondaar et al. 1995); from Varshets (Bulgaria) as Muscicapa sp. (Boev 1996, 2000); from Mas Ramboult (France) as Ficedula hypoleuca (Pallas, 1764) (Mourer-Chauviré 1975) and as Ficedula sp. from Montoussé (France) (Clot et al. 1976).

\section{- Erithacus Cuvier, 1801}

\section{- Erithacus † horusitskyi Kessler et Hír, 2012}

Type locality and age: Mátraszőlós 1, Middle Miocene (MN 7-8) (Hungary) (Kessler \& Hír 2012). In size, it is similar to Erithacus and Saxicola species, except for size of the length of proximal epiphysis, while in shape it is more similar to Erithacus. Previously was reported as Parus sp. (Gál et al. 1999).

- Erithacus $\uparrow$ minor Kessler, 2013

Type locality and age: Beremend 26, Pliocene (MN 15) (Hungary) (Kessler 2013a, 2013b). It corresponds in characteristics to extant species, but it is smaller.

\section{- Erithacus rubecula (Linnaeus, 1758)}

Q1: Betfia 2, 9 (Romania) (Kormos 1913, Čapek 1917, Lambrecht 1933, Kesssler 1975, Jánossy 1979, Gál 2002); Q3/I: Hundsheim (Austria) (Mlíkovskỳ 2009); Q4/I: Velika Pecina (Croatia) (V. Malez 1975, 1984, 1988); Q4/II: Hosszúhegyi Cave (Hungary) (Jánossy 1979); Szegyestel-völgyi Caves (Peșteri din Valea Sighiștelului) (Romania) (Kessler 1982). From sites in Europe outside the Carpathian Basin Q1-2: Spain; Q3: France, Italy, United Kingdom; Q4: Austria, Croatia, France, Georgia, Germany, Greece, Ireland, Italy, Spain, Serbia, Switzerland, Ukraine, United Kingdom (Tyrberg 1998).

- Erithacus sp.

Q1: Beremend 17 (Jánossy 1992, 1995).

\section{- Luscinia Forster, 1817}

\section{- Luscinia $\uparrow$ praeluscinia Kessler et Hír, 2012}

Type locality and age: Litke 2, Lower Miocene (MN 5) (Hungary) (Kessler \& Hír 2012). In size, it is closest to the extant Luscinia luscinia.

\section{- Luscinia $\uparrow$ jurcsaki Kessler et Venczel, 2011}

Type locality and age: Köalja 2 (Subpiatra) (Romania), Middle Miocene (MN 6) (Hungary) (Kessler \& Venczel 2011).

The distal fragment of the femur typically refers to the family of flycatchers (Muscicapidae). It was previously reported as such (Kessler \& Venczel 2009). Within this, it differs in size from the larger genus Muscicapa, Saxicola, Erithacus, Phoenicurus and much smaller than the genus Oenanthe and Monticola.

\section{- Luscinia $\uparrow$ denesi Kessler, 2013}


Type locality and age: Polgárdi 4, Upper Miocene (MN 13) (Hungary) (Kessler 2013a, 2013b). It corresponds in characteristics to the extant genus. Previously was reported as Luscinia sp. (Jánossy 1991, 1995).

\section{- Luscinia † pliocaenica Kessler, 2013}

Type locality and age: Beremend 26, Pliocene (MN 15) (Hungary) (Kessler 2013a, 2013b). The characteristics of bones correspond to extant genus despite the damages; however, they have larger dimensions than in extant species.

\section{- Luscinia luscinia (Linnaeus, 1758)}

Q1: Betfia 9 (Romania) (Gál 2002). From sites in Europe outside the Carpathian Basin Q3: France; Q4: Austria, Germany, Italy, Spain, United Kingdom (Tyrberg 1998).

\section{- Luscinia megarhynchos C. L. Brehm, 1831}

Q1: Betfia 9 (Romania) (Gál 2002); Q4/II: Bodajk-Rigólyuk (Hungary) (Kordos 1984). From sites in Europe outside the Carpathian Basin Q4: Spain, Ukraine, United Kingdom (Tyrberg 1998).

\section{- Luscinia svecica (Linnaeus, 1758)}

Q3/I: Hundsheim (Austria) (Mlíkovskỳ 2009). From sites in Europe outside the Carpathian Basin Q4: Germany (Tyrberg 1998).

\section{- Luscinia sp.}

Q1: Németóvár 4B (Deutsch-Altenburg) (Austria) (Jánossy 1981) (after Mlikovskỳ 1998 is Sylvia atricapilla). From sites in Europe outside the Carpathian Basin Q4: Germany (Tyrberg 1998).

The genus was reported outside the Carpathian Basin as Luscinia svecica (Linnaeus, 1758) by Jánossy from Rebielice I. (Upper Pliocene, Poland) (Jánossy 1974) and from Stránská skála (Q1, Czech Republic) (Jánossy 1972).

\section{- Saxicola Bechstein, 1892}

\section{- Saxicola $\dagger$ lambrechti Kessler, 2013}

Type locality and age: Polgárdi 4, 5; Upper Miocene (MN 13) (Hungary) (Kessler 2013a, 2013b). It corresponds in characteristics and sizes to extant species of the genus.

\section{- Saxicola $\dagger$ baranensis Kessler, 2013}

Type locality and age: Csarnóta 2, Pliocene (MN 15) (Hungary) (Kessler 2013a, 2013b). Other locality and age: Beremend 26, Pliocene (MN 15) (Hungary) (Kessler 2013a, 2013b). Its characteristic corresponds to extant genus and has larger sizes than extant species.

\section{- Saxicola † parva Kessler, 2013}

Type locality and age: Csarnóta 2, Pliocene (MN 15) (Kessler 2013a, 2013b). It is smaller than $S$. baranensis. The characteristics and dimensions correspond to smaller species of the extant genus. It is smaller than $S$. baranensis.

\section{- Saxicola $\uparrow$ magna Kessler, 2013}

Type locality and age: Beremend 26, Pliocene (MN 15) (Hungary) (Kessler 2013a, 2013b). Its characteristic corresponds to extant genus and has larger sizes than extant species.

- Saxicola rubetra (Linnaeus, 1758)

Q1: Betfia 9 (Romania) (Gál 2002); Q4/II: Körösbánlaki Cave (Romania) (Kessler 1982). From sites in Europe outside the Carpathian Basin Q3: Czech Republic, France, Spain; Q4: Czech Republic, France, Ireland, Russia, Ukraine, United Kingdom (Tyrberg 1998). 


\section{- Saxicola torquata (Linnaeus, 1766)}

Q1: Betfia 2 (Romania) (Kormos 1913, Čapek 1917, Lambrecht 1933, Kessler 1975, Jánossy 1979, Gál 2002); Q4/I: Gencsapáti (Hungary) (Jánossy 1979). From sites in Europe outside the Carpathian Basin Q4: Germany (Tyrberg).

The genus is known outside the Carpathian Basin more from Early Pleistocene (Q1) from Voigstedt (Germany) (Jánossy 1965) and from Quibas (Spain) (Montoya et al. 1999).

- Monticola Boie, 1822

- Monticola $\uparrow$ pongraczi Kessler, 2013

Type locality and age: Beremend 26, Pliocene (MN 15) (Hungary) (Kessler 2013a, 2013b). It corresponds mostly in characteristics and size to extant species.

- Monticola saxatilis (Linnaeus, 1766)

Q4/I: Barcarozsnyó Gura Cheii Cave (Peștera Gura Cheii-Râşnov) (Romania) (Gál 1998, 2002). The genus is known only the Middle and the Late Pleistocene to extant species from France, Spain (Tyrberg 1998).

\section{- Monticola solitarius (Linnaeus, 1758)}

The extant species is known only outside of Carpathian Basin Q3: France; Q4: France, Greece, Spain (Tyrberg 1998).

- Phoenicurus Forster, 1817

\section{- Phoenicurus $\uparrow$ erikai Kessler, 2013}

Type locality and age: Csarnóta 2, Pliocene (MN 15) (Hungary) (Kessler 2013a, 2013b). It corresponds in characteristics to extant genus, but it is similar in size to smaller extant species.

\section{- Phoenicurus $\uparrow$ baranensis Kessler, 2013}

Type locality and age: Beremend 26, Pliocene (MN 15) (Hungary) (Kessler 2013a, 2013b). Its characteristic corresponds to the extant genus but has larger dimensions.

\section{- Phoenicurus phoenicurus (Linnaeus, 1758)}

Q3/I: Hundsheim (Austria) (Jánossy 1974a). From sites in Europe outside the Carpathian Basin Q3: France, Germany; Q4: Czech Republic, France, Germany, Russia, Ukraine, United Kingdom (Tyrberg 1998).

\section{- Phoenicurus ochrurus (Gmelin, 1789)}

Q4/II: Grosse Offenbergerhöhle (Austria) (Bocheński \& Tomek 1994).

The genus was reported only from Quibas - Spain (Lower Pleistocene, Q1) (Montoya et al. 1999).

\section{- Oenanthe Viellot, 1816}

- Oenanthe $\uparrow$ kormosi Kessler, 2013

Type locality and age: Polgárdi 4, Upper Miocene (MN 13) (Hungary) (Kessler 2013a, 2013b). In its characteristics and sizes close to the extant species Oenanthe oenanthe (Linnaeus, 1758) but is somewhat larger than that and alsoother species inthe family, but it is smaller than Monticola.

\section{- Oenanthe † pongraczi Kessler, 2013}

Type locality and age: Csarnóta 2, Pliocene (MN 15) (Hungary) (Kessler 2013a, 2013b). Other locality and age: Beremend 26, Pliocene (MN 15) (Hungary) (Kessler 2013a, 2013b). It mostly corresponds in charachteristics to extant species Oenanthe oenanthe but is larger. 


\section{- Oenanthe oenanthe (Linnaeus, 1758)}

Q3/I: Hundsheim (Austria) (Lambrecht 1933, Jánossy 1979); Q4/II: Grosse Offenbergerhöhle (Austria) (Bocheński \& Tomek 1994); Hosszúhegyi Cave (Hungary) (Jánossy 1979).

- Oenanthe hispanica (Linnaeus, 1758)

The extant species was reported outside of Carpathian Basin from Q3: France; Q4: Greece (Tyrberg 1998).

- Oenanthe leucura (J. F. Gmelin, 1789)

Q4: France (Tyrberg 1998).

The earliest report of the genus outside the Carpathian Basin is only the Early Pleistocene (Q1) from Stránská skála (Czech Republic) (Jánossy 1972); Montoussé 5. (France) (Clot et al. 1976); Quibas (Spain) (Montaya et al. 1999).

The fossil species indicated here from Polgárdi, Csarnóta 2 and Beremend 26 was reported previously as Muscicapidae sp. foss. indet. (Kessler 2010).

\section{Fam. Turdidae Rafinesque, 1815}

$-\uparrow$ Turdicus Kretzoi, 1962

- †Turdicus matraensis Kessler et Hír, 2012

Type locality and age: Mátraszőlős 3, Middle Miocene (MN 7-8) (Hungary) (Kessler \& Hír 2012).

Their features are partly consistent with the new genus described by Miklós Kretzoi from the Betfia 5 (Lower Pleistocene) site by a left coracoid (1962), as the bone is more graceful (?) than the extant species. However, it differs in size from the genus type species (Turdicus tenuis Kretzoi, 1962), which is similar in size to that of the Mistle Thrush (Turdus viscivorus).

\section{$-\uparrow$ Turdicus pannonicus Kessler, 2013}

Type locality and age: Polgárdi, Upper Miocene (MN 13) (Hungary) (Kessler 2013a, 2013b). It presents morphological characteristics corresponding to the genus and intermediate dimensions between Turdicus matraensis and T. tenuis.

\section{$-\uparrow$ Turdicus tenuis Kretzoi, 1962}

Type locality and age: Betfia 5, Q2 (Romania) (Kretzoi 1962).

The original diagnosis is that it is typically a gracillary bone. Unfortunately, the holotype has been lost, and image-size and dimensions have not been reported, so it is considered as 'nomen nudum' (Brodkorb 1978, Mlikovskỳ 2002).

The fossil genus has not yet been identified from the area outside the Carpathian Basin, but here it is continuously present from the Middle Miocene to the Lower Pleistocene.

- Turdoides Cretzschmar 1826

- Turdoides $\dagger$ borealis Jánossy, 1979

Type localities and age: Csarnóta 2, Pliocene (MN 15) (Hungary) (Jánossy 1979); other locality and age: Osztramos 1, Pliocene (MN 16) (Hungary) (Jánossy 1979). It is smaller in size than Turdus philomelos and T. iliacus. The genus had not been labeled elsewhere from fossil material.

- Turdus Linnaeus, 1758

- Turdus $\uparrow$ miocaenicus Kessler, 2013 
Type locality and age: Polgárdi 5, Upper Miocene (MN 13) (Hungary) (Kessler 2013a, 2013b). Its size and characteristics are very similar to extant larger trushes' (Turdus pilaris/ viscivorus/torquatus) size.

\section{- Turdus † polgardiensis Kessler, 2013}

Type locality and age: Polgárdi 5, Upper Miocene (MN 13) (Hungary) (Kessler 2013a, 2013b). In size, it is similar to medium-size thrushes (Turdus merula). In the morphological characteristics, it is more similar to larger species of the genus.

\section{- Turdus † major Kessler, 2013}

Type locality and age: Csarnóta 2, Pliocene (MN 15) (Hungary) (Kessler 2013a, 2013b). Its characteristics are similar to extant species and has the size of $T$. torquatus.

\section{- Turdus † medius Kessler, 2013}

Type locality and age: Csarnóta 2, Pliocene (MN 15) (Hungary) (Kessler 2013a, 2013b). It corresponds in characteristies to extant genus, and in dimensions to Turdus merula.

- Turdus † praeminor Kessler, 2019 / syn: Turdus minor Kessler 2013

Type locality and age: Csarnóta 2, Pliocene (MN 15) (Hungary) (Kessler 2013a, 2013b). Other locality and age: Beremend 26, Pliocene (MN 15) (Hungary) (Kessler 2013a, 2013b). It corresponds in characteristies to extant genus, and in dimensions to Turdus philomelos, but it is smaller. Originally was named as T. minor, but since the name is already reserved for one of the extant species in the Bahamas Islands, it has been renamed.

\section{- Turdus torquatus Linnaeus, 1758}

Q1: Betfia 9 (Romania) (Gál 2002); Q4/I: Körösmart (Rîpa) (Romania) (Jánossy in Hamar \& Csák 1969, Kessler 1974a, Gál 2002); Q4/II: Teufelslucke (Austria) (Soergel 1966). From sites in Europe outside the Carpathian Basin; Q3: France, Germany, Spain; Q4: Austria, Bulgaria, Croatia, Czech Republic, France, Germany, Italy, Poland, Spain, United Kingdom (Tyrberg 1998).

\section{- Turdus merula Linnaeus, 1758}

Q1: Betfia 2 (Kormos 1913, Čapek 1917, Lambrecht 1933, Kessler 1975, Jánossy 1979, Gál 2002), Betfia 9 (Kessler 1975, Gál 2002) (all in Romania); Q3/I: Tarkő 1 (Hungary) (Jánossy 1979); Betfia 7/4 (Romania) (Kessler 1975, Jánossy 1979, Gál 2002); Q4/I: Krapina (V. Malez 1973, 1984, V. Malez-Bačić 1975), Velika Pecina (V. Malez 1975, 1984, 1988), Veternica (V. Malez 1973, 1988, V. Malez-Bačić 1975) (all in Croatia); Tatabánya-Kálváriahegy 4. Cave (Hungary) (Gál 2005a, 2005b); Körösmart (Rîpa) (Jánossy in Hamar \& Csák 1969, Kessler 1974a, Gál 2002), Ohábaponor-Bordu Mare Cave (Ohaba Ponor-Peştera Bordu Mare) (Kessler 1985, Jurcsák \& Kessler 1988, Gál 2002, 2003), Szegyestel-Măgura Cave (Sighiştel, Peştera Măgura), Szegyestel-Tibocoaia Cave (Sighiștel) (Kessler 1982, 1985, Gál 2002) (all in Romania); Q4/II: Teufelslucke (Austria) (Soergel 1966); Ecsegfalva (Pike-Tay et al. 2004, Gál 2007), Legény Cave (Kormos 1914), Miskolc-Névtelen Cave (Kessler 2010) (all in Hungary); Körösbánlaki Cave (Peştera din Bălnaca) (Kessler 1982), Püspökfürdő Lake (lacul din Băile Episcopești) (Kessler 1974b, 1985), Révi Caves (Peşterile din Vadu Crișului), Szegyestel-Drăcoaia Cave (Sighiştel, Peştera Drăcoaia), Szegyestelvölgyi Caves (Peșteri din Valea Sighiștelului) (Kessler 1982), Szkerisoara-Coiba Mare Cave (Scărişoara, Peştera Coiba Mare) (Kessler 1982, Jurcsák \& Kessler 1986, 1988), Vargyasi-szoros - Homoródalmási Caves (Peșteri din Defileul Vârghișului) (Jurcsák \& Kessler 
1986, 1988) (all in Romania). From sites in Europe outside the Carpathian Basin Q1-2: France; Q3: Azerbaijan, Czech Republic, France, Italy, Spain, Ukraine, United Kingdom; Q4: Austria, Bosnia-Herzegovina, Bulgaria, Croatia, Czech Republic, France, Georgia, Germany, Ireland, Italy, Luxemburg, Montenegro, Moldova, Poland, Serbia, Spain, Ukraine, United Kingdom (Tyrberg 1998).

\section{- Turdus philomelos C. L. Brehm, 1831}

Q1: Németóvár (Austria) (Jánossy 1981); Beremend 16 (Hungary) (Jánossy 1992); Betfia 2 (Kormos 1913, Čapek 1917, Lambrecht 1933, Kessler 1975, Jánossy 1979, Gál 2002), Betfia 9 (Kessler 1975, Gál 2002) (all in Romania); Q3/I: Hundsheim (Austria) (Lambrecht 1933, Jánossy 1974a, 1979); Süttö 1-4 (Hungary) (Jánossy 1979); Q4/I: Merkenstein (Austria) (Wettstein \& Mühlhofer 1938); Budapest-Remetehegyi Niche (Kormos 1914, Lambrecht 1933, Jánossy 1979, 1986), Hámor-Herman Ottó Cave (Lambrecht 1915, 1933), Pilisszántói I. Niche (Lambrecht 1915, 1933, Jánossy 1979, 1986) (all in Hungary); Barcarozsnyó (Peștera Gura Cheii-Râşnov) (Gál 1998, 2002), Szegyestel-Măgura Cave (Sighiştel, Peştera Măgura), Szegyestel-Tibocoaia Cave (Sighiștel, Peştera Tibocoaia) (Kessler 1982, 1985, Gál 2002) (all in Romania); Óruzsin-Antal Cave (Oruzer) (Slovakia) (Nehring 1880, Róth 1881, Lambrecht 1912, 1933); Q4/II: Legény Cave (Kormos 1914), Ordacsehi-Kistöltés (Gál 2004, 2005b) (all in Hungary); Peterd-Tordai Defile, Magyar Cave (Petrești, Cheile Turzii-Peștera Ungurească) (Kessler \& Gál 1998, Gál 2005b), Remetelórév-Bólyikői Cave (Lorău-Peştera din Piatra Boiului) (Kessler 1982), Révtizfalusi Cave (peştera din Zece Hotare) (Kessler 1985), Szegyestel-Drăcoaia Cave (Sighiştel, Peştera Drăcoaia), Szegyestel-völgyi Caves (Peşteri din Valea Sighiştelului) (Kessler 1982), Vársonkolyosi Caves (peşteri din (Şuncuiuș)) (Kessler 1977, Gál 2002) (all in Romania). From sites in Europe outside the Carpathian Basin Q1-2: Bulgaria; Q3: Ukraine; Q4: Belgium, Bulgaria, Croatia, Czech Republic, France, Georgia, Germany, Ireland, Italy, Portugal, Poland, Spain, Switzerland, Ukraine, United Kingdom (Tyrberg 1998).

\section{- Turdus iliacus Linnaeus, 1766}

Q1: Betfia 2 (Kormos 1913, Čapek 1917, Lambrecht 1933, Kessler 1975, Jánossy 1979, Gál 2002), Betfia 9 (Kessler 1975, Gál 2002) (all in Romania); Q2: Nagyharsányhegy 1-4 (Hungary) (Kessler 2010); Q3/I: Hundsheim (Austria) (Lambrecht 1933, Jánossy 1979); Q4/I: Barcarozsnyó (Romania) (Gál 1998, 2002); Hámor-Puskaporos (Lambrecht 1912, 1916, 1933, Jánossy 1979, 1986), Szilvásvárad-Istállóskői Cave (Lambrecht 1912, 1933, Jánossy 1952, 1955, 1979, 1986) (all in Hungary); Barcarozsnyó - Gura Cheii Cave (Peștera Gura Cheii-Râşnov) (Romania) (Gál 1998, 2002); Q4/II: Teufelslucke (Austria) (Soergel 1966). From sites in Europe outside the Carpathian Basin Q1-2: France, Spain; Q3: Azerbaijan, France, Italy, Spain, Ukraine, United Kingdom; Q4: Austria, Belgium, Czech Republic, France, Greece, Germany, Ireland, Italy, Portugal, Poland, Spain, Switzerland, Ukraine, United Kingdom (Tyrberg 1998).

\section{- Turdus iliacus / T. musicus (=philomelos)}

Q4/I: Szilvásvárad-Istállóskői Cave (Lambrecht 1912, 1933, Jánossy 1952, 1955, 1979, 1986) (all in Hungary);

\section{- Turdus viscivorus / T. torquatus}

Q4/I: Varbó-Lambrecht Kálmán Cave (Hungary) (Jánossy 1964,1979); 


\section{- Turdus viscivorus Linnaeus, 1758}

Q1: Németóvár (Deutsch-Altenburg) (Austria) (Jánossy 1981); Betfia 2 (Kormos 1913, Čapek 1917, Lambrecht 1933, Kessler 1975, Jánossy 1979, Gál 2002), Betfia 9 (Kessler 1975, Gál 2002) (all in Romania); Q3/I: Hundsheim (Lambrecht 1933, Jánossy 1974a, 1979), Merkenstein (Wettstein \& Mühlhofer 1938) (all in Austria); Vindija (Croatia) (M. Malez 1961, V. Malez 1973, 1988); Q4/I: Krapina (Lambrecht 1915, V. Malez 1973, 1984), Velika pec na Lipi (V. Malez 1975, 1984, V. Malez-Bačić 1975, 1979) (all in Croatia); Budapest-Remetehegyi Niche (Kormos 1914, Lambrecht 1933, Jánossy 1979, 1986), Csobánka-Kiskevélyi Cave (Lambrecht 1912，1915, 1933, Jánossy 1979), Felsőtárkány-Peskő Cave (Lambrecht 1912, 1933, Jánossy 1979, 1986), Hámor-Puskaporos Niche (Lambrecht 1912, 1916, 1933, Jánossy 1979, 1986), Hámor-Herman Ottó Cave (Lambrecht 1915, 1933), Pilisszántói I. Niche (Lambrecht 1915, 1933, Jánossy 1979, 1986), Répáshuta-Balla Cave (Lambrecht 1912, 1933), Szilvásvárad-Istállóskői Cave (Lambrecht 1912, 1933, Jánossy 1952, 1955, 1979, 1986) (all in Hungary); Barcarozsnyó-Gura Cheii Cave (Peștera Gura Cheii-Râşnov) (Gál 1998, 2002), Ohábaponor-Bordu Mare Cave (Ohaba Ponor-Peştera Bordu Mare) (Kessler 1985, Jurcsák \& Kessler 1988, Gál 2002, 2003), Szegyestel-Măgura Cave, Szegyestel-Tibocoaia Cave (Peștera Tibocoaia) (Sighiștel) (Kessler 1982, 1985, Gál 2002) (all in Romania); Detrekőszentmiklós-Pálffy Cave (Dzeráva Skála-Plavecky Mikulas) (Slovakia) (Lambrecht 1913, 1933); Q4/II: Teufelslucke (Austria) (Soergel 1966); Ecsegfalva (Pike-Tay et al. 2004, Gál 2007), Felsőtárkány-Petényi Cave (Jánossy 1979), Hosszúhegyi Cave (Jánossy 1979) (all in Hungary); Herkulesfürdő-Rablók Cave (Băile Herculane, Peştera Hoţilor) (Kessler 1981, Gál 2002), Körösbánlaki Cave (Peştera din Bălnaca) (Kessler 1982), Peterd-Tordai Defile - Magyar Cave (Petrești, Cheile Turzii-Peștera Ungurească) (Kessler \& Gál 1998, Gál 2005a), Révi Caves (Peşterile din Vadu Crișului) (Kessler 1982), Szegyestel-Drăcoaia Cave (Sighiştel, Peştera Drăcoaia) (Kessler 1982), Szkerisoara-Sasok Cave (Scărişoara, Peştera Vulturilor) (Kessler 1982, Jurcsák \& Kessler 1986, 1988), Vársonkolyos-Izbîndiş Cave, Vársonkolyos-Kis Magyar Cave (Şuncuiuş, Peştera Izbîndiş; Peştera Napiştileu),Vársonkolyosi Caves (Şuncuiuş) (Kessler 1977, Gál 2002) (all in Romania). From sites in Europe outside the Carpathian Basin Q1-2: France, Spain; Q3: Czech Republic, France, Italy, Russia, Spain; Q4: Belgium, Croatia, Czech Republic, France, Germany, Greece, Ireland, Italy, Moldova, Poland, Russia, Spain, Ukraine, United Kingdom (Tyrberg 1998).

\section{- Turdus pilaris Linnaeus, 1758}

Q1: Betfia 2 (Kormos 1913, Čapek 1917, Lambrecht 1933, Kessler 1975, Jánossy 1979, Gál 2002), Betfia 9 (Kessler 1975, Gál 2002) (all in Romania); Q3/II: Süttő 1-4 (Hungary) (Jánossy 1979); Q4/I: Bajót-Öregkő (Kormos 1914), Budapest-Remetehegyi Niche (Kormos 1914, Lambrecht 1933, Jánossy 1979, 1986), Felsőtárkány-Peskő Cave (Lambrecht 1912, 1933, Jánossy 1979, 1986), Hámor-Puskaporos Niche (Lambrecht 1912, 1916, 1933 , Jánossy 1979, 1986), Pilisszántói I. Niche (Lambrecht 1915, 1933, Jánossy 1979, 1986), Répáshuta-Balla Cave (Lambrecht 1912, 1933), Szilvásvárad-Istállóskői Cave (Lambrecht 1912, 1933, Jánossy 1952, 1955, 1979, 1986) (all in Hungary); Hidegszamos-Csont Cave (Someşul Rece-Peştera cu Oase) (Lambrecht 1915), Körösmart (Rîpa) (Jánossy in Hamar \& Csák 1969, Kessler 1974a, Gál 2002), Nándor-Nándori Cave (Nandru-Peştera 
Curata) (Jánossy 1965, Fischer \& Stephan 1977, Kessler 1985, Jurcsák \& Kessler 1988, Gál 2002, 2003), Ohábaponor-Bordu Mare Cave (Ohaba Ponor-Peştera Bordu Mare) (Kessler 1985, Jurcsák \& Kessler 1988, Gál 2002, 2003), Szegyestel-Măgura Cave (Sighiştel, Peştera Măgura) (Kessler 1982, 1985, Gál 2002) (all in Romania); Óruzsin-Antal Cave (Oruzer) (Slovakia) (Nehring 1880, Róth 1881, Lambrecht 1912, 1933); Q4/II: Teufelslucke (Austria) (Soergel 1966); Felsőtárkány-Petényi Cave (Jánossy 1979); Legény Cave (Lambrecht 1914), Répáshuta-Rejteki Niche (Jánossy 1962, 1979, 1986) (all in Hungary); Peterd-Tordai-Defile - Magyar Cave (Petrești, Cheile Turzii-Peștera Ungurească) (Kessler \& Gál 1998, Gál 2004), Szegyestel-Drăcoaia Cave (Sighiştel, Peştera Drăcoaia), Szegyestel-völgyi Caves (peşteri din Valea Sighiştelului) (Kessler 1982), Szkerisoara-Sasok Cave (Scărişoara, Peştera Vulturilor).

(Kessler 1982, Jurcsák \& Kessler 1986, 1988), Vargyasi-szoros - Homoródalmási Caves (peșteri din Defileul Vârghiṣului) (Jurcsák \& Kessler 1986, 1988), Vársonkolyos-Izbîndiş Cave (Şuncuiuş, Peştera Izbîndiş̧) (Kessler 1977, Gál 2002) (all in Romania). From sites in Europe outside the Carpathian Basin Q3: Czech Republic, France, Germany, Italy, Spain; Q4: Austria, Belgium, Croatia, Czech Republic, France, Germany, Greece, Ireland, Italy, Portugal, Russia, Spain, Switzerland, Ukraine, United Kingdom (Tyrberg 1998).

\section{- Turdus pilaris / T. merula}

Q4/I: Szamosfalva (Someșeni) (Romania) (Kormos 1913, Lambrecht 1933);

\section{- Turdus sp. foss. indet.}

MN 7-8: Felsőtárkány (Hungary) (Hír et al. 2001); MN 13: Polgárdi 4 (Hungary) (Jánossy 1991, 1995 - as: Turdus iliacus); MN 15: Ivánháza I (Ivanovce I) (Slovakia) (Švec in Fejfar \& Heinrich 1985, Mlíkovskỳ 2002); MN 15: Csarnóta 2 (Jánossy 1979 - as: Turdus viscivorus), Beremend 26 (Kessler 2010) (all in Hungary); MN 16: Betfia 13 (Romania) (Kessler 1975, Gál 2002);

\section{- Turdus sp. indet.}

Q1: Németóvár 4B (Deutsch-Altenburg) (Austria) (Jánossy 1981, Döppes \& Rabeder 1997, Mlíkovskỳ 1998); Beremend 17 (Hungary) (Kessler 2010); Betfia 9 (Romania) (Gál 2002); Q2: Nagyharsányhegy 1-4 (Hungary) (Lambrecht 1916, 1933, Jánossy 1979); Q3/I: Hundsheim (Austria) (Mlíkovskỳ 2009); Q3/II: Süttő 1-4 (Hungary) (Kessler 2009); Q4/I: Csobánka-Kiskevélyi Cave (Lambrecht 1912, 1915, 1933, Jánossy 1979), FelsőtárkányPeskő Cave (Lambrecht 1912, 1933, Jánossy 1979, 1986), Hámor-Puskaporos Niche (Lambrecht 1912, 1916, 1933, Jánossy 1979, 1986), Száraz-Gerence (Jánossy 1979, 1986) (all in Hungary); Körösmart (Rîpa) (Romania) (Jánossy in Hamar \& Csák 1969, Kessler 1974a, Gál 2002). From sites in Europe outside the Carpathian Basin Q1-2: Croatia, Germany; Q3: Czech Republic, Gerorgia, Grece, Germany, Italy, Poland, Spain, Turkey; Q4: Austria, Belgium, Bosnia-Herczegovina, Bulgaria, Croatia, Czech Republic, France, Georgia, Germany, Greece, Ireland, Italy, Malta, Moldova, Poland, Russia, Spain, Switzerland, Ukraine, United Kingdom (Tyrberg 1998).

The genus it is known outside of the Carpathian Basin from Credinta - Romania (Middle Miocene, MN 8) as Turdus sp. (Gál \& Kessler 2006), while from the Late Pliocene from Rebielice Królowskie I. - Poland (Jánossy 1974b), Varshets-Bulgaria (Boev 1996, 2000), Sandalja I. - Croatia (V. Malez-Bacic 1979). 


\section{Fam. Oriolidae Boie, 1826}

- Oriolus Linnaeus, 1758

- Oriolus † beremendensis Kessler, 2013

Type locality and age: Beremend 26, Pliocene (MN 15) (Hungary) (Kessler 2013a, 2013b). It corresponds partially with charateristics and size of the extant species.

- Oriolus oriolus (Linnaeus, 1758)

Q1: Betfia 9 (Romania) (Gál 2002); Q3: Vindija (V. Malez 1973, 1988) (Croatia); Q4/I: Budapest-Remetehegyi Niche (Kormos 1914, Lambrecht 1933, Jánossy 1979, 1986), Pilisszántói I. Niche (Lambrecht 1915, 1933, Jánossy 1979, 1986) (all in Hungary); Q4/II: Peterd-Tordai-Defile - Magyar Cave (Petrești, Cheile Turzii-Peștera Ungurească) (Kessler \& Gál 1998, Gál 2005a), Révi Caves (Peşterile din Vadu Crișului) (Kessler 1982) (all in Romania).

The family and genus have no other extinct species. The extant species Oriolus oriolus is known from some localities from the Late Pleistocene (Q4) in Europa: Bosnia-Herczegovina, Croatia, Czech Republic, France, Germany, Italy (Tyrberg 1998).

\section{Fam. Sylviidae Vigors, 1825}

- Acrocephalus Naumann, 1811

- Acrocephalus $\uparrow$ major Kessler, 2013

Type locality and age: Polgárdi 5, Upper Miocene (MN 13) (Hungary) (Kessler 2013a, 2013b). Corresponds in its characteristics and sizes to extant larger species of the genus.

\section{- Acrocephalus $\uparrow$ minor Kessler, 2013}

Type locality and age: Polgárdi 5, Upper Miocene (MN 13) (Hungary) (Kessler 2013a, 2013b). Corresponds in characteristic to smaller species of the genus. The fosil species from Polgárdi was reported previously as Acrocephalus sp. by Jánossy $(1991,1995)$.

\section{- Acrocephalus $\uparrow$ kretzoii Kessler, 2013}

Type locality and age: Csarnóta 2, Pliocene (MN 15) (Hungary) (Kessler 2013a, 2013b). Other locality and age: Beremend 26, Pliocene (MN 15) (Hungary) (Kessler 2013a, 2013b). Its characteristics correspond to extant genus but in dimensions more similar to a larger species.

\section{- Acrocephalus $\uparrow$ kordosi Kessler, 2013}

Type locality and age: Csarnóta 2, Pliocene (MN 15) (Hungary) (Kessler 2013a, 2013b). Its characteristics correspond to extant genus, but its dimensions place it among the smaller species.

- Acrocephalus sp. foss. indet.

MN 9: Rudabánya (Hungary) (Jánossy 1993); MN 16: Beremend 26 (Hungary) (Kessler 2010); Q1: Betfia 9 (Romania) (Gál 2002).

- Acrocephalus palustris (Bechstein, 1811)

Q1: Betfia 2 (Romania) (Kormos 1913, Čapek 1917, Lambrecht 1933, Kessler 1975, Jánossy 1979, Gál 2002). From sites in Europe outside the Carpathian Basin Q4: France (Tyrberg 1998).

\section{- Acrocephalus paludicola (Viellot, 1817)}

From sites in Europe outside the Carpathian Basin Q4: France (Tyrberg 1998). 


\section{- Acrocephalus schoenobaenus (Linnaeus, 1758)}

From sites in Europe outside the Carpathian Basin Q4: United Kingdom (Tyrberg 1998).

- Acrocephalus scirpaceus (Hermann, 1894)

From sites in Europe outside the Carpathian Basin Q4: Spain (Tyrberg 1998).

- Acrocephalus arundinaceus (Linnaeus, 1758)

From sites in Europe outside the Carpathian Basin Q3: France; Q4: Austria, France, Romania (Tyrberg 1998).

- Acrocephalus sp.

Q4/II: Ecsegfalva (Hungary) (Pike-Tay et al. 2004, Gál 2007).

From sites in Europe outside the Carpathian Basin Q3: Czech Republic Q4: France (Tyrberg 1998).

- Cettia Bonaparte, 1838

- Cettia † janossyi Kessler, 2013

Type locality and age: Polgárdi 5, Upper Miocene (MN 13) (Hungary) (Kessler 2013a, 2013b). Corresponds in its characteristics and sizes to extant species. This material was reported previously as Cettia sp. by Jánossy (1991).

- Cettia $\uparrow$ kalmani Kessler, 2013

Type locality and age: Csarnóta 2, Pliocene (MN 15) (Hungary) (Kessler 2013a, 2013b). Its characteristics correspond to extant species, but the fossil species is bigger in sizes than extinct species from Polgárdi Cettia janossyi or than the extant species. The genus was reported only from the Carpathian Basin.

- Hippolais C. von Baldenstein, 1827

- Hippolais $\uparrow$ veterior Kessler, 2013

Type locality and age: Polgárdi 5, Upper Miocene (MN 13) (Hungary) (Kessler 2013a, 2013b). Its characteristics mostly correspond to those of extant genus.

- Hippolais sp. foss. indet.

MN 15: Csarnóta 2 (Hungary) (Jánossy 1979); Q1: Betfia 2 (Romania) (Kormos 1913, Čapek 1917, Lambrecht 1933, Kessler 1975, Jánossy 1979, Gál 2002). The genus was reported outside the Carpathian basin only from France (Upper Pleistocene, Q4) with extant species Hippolais icterina (Vieillot, 1817) (Tyrberg 1998).

- Sylvia Scopoli, 1769

- Sylvia † intermedia Kessler, 2013

Type locality and age: Polgárdi 5, Upper Miocene (MN 13) (Hungary) (Kessler 2013a, 2013b). The bones belong to medium-size species. The fossil material was reported previously as Sylvia sp. by Jánossy (1991).

- Sylvia † pusilla Kessler, 2013

Type locality and age: Csarnóta 2, Pliocene (MN 15) (Hungary) (Kessler 2013a, 2013b). Other locality and age: Beremend 26, Pliocene (MN 15) (Hungary) (Kessler 2013a, 2013b). The bones belong to one smaller species.

- Sylvia atricapilla (Linnaeus, 1758)

Q1: Betfia 9 (Romania) (Gál 2002); Németóvár (Austria) (Jánossy 1981). From sites in Europe outside the Carpathian Basin Q1-2: Spain; Q3: France; Q4: Czech Republic, France, Spain, United Kingdom (Tyrberg 1998). 


\section{- Sylvia communis Latham, 1787}

Q1: Betfia 2 (Kormos 1913, Čapek 1917, Lambrecht 1933, Kessler 1975, Jánossy 1979, Gál 2002), Betfia 9 (Kessler 1975, Gál 2002) (all in Romania). From sites in Europe outside the Carpathian Basin Q3: France; Q4: France (Tyrberg 1998).

- Sylvia borin (Boddaert, 1783)

Q3: Vindija (Croatia) (M. Malez 1961, V. Malez 1973, 1988, M. Malez \& Rukavina 1979); Q4/I: Velika pec na Lipi (Croatia) (V. Malez 1975, 1984, V. Malez-Bačić 1975, 1979). From sites in Europe outside the Carpathian Basin Q3: United Kingdom; Q4: Croatia (Tyrberg 1998).

- Sylvia curruca (Linnaeus, 1758)

Q4/I: Óruzsin-Antal Cave (Oruzer, Antal Cave, Slovakia) (Nehring 1880, Róth 1881, Lambrecht 1912, 1933). From sites in Europe outside the Carpathian Basin Q4: Russia (Tyrberg 1998).

- Sylvia melanocephala (J. F. Gmelin, 1788)

From sites in Europe outside the Carpathian Basin Q3: France (Tyrberg 1998).

- Sylvia hortensis (J. F. Gmelin, 1788)

From sites in Europe outside the Carpathian Basin Q4: France (Tyrberg 1998).

- Sylvia nisoria (Bechstein, 1785)

From sites in Europe outside the Carpathian Basin Q3: France (Tyrberg 1998).

- Sylvia sp. foss. indet.

MN 15: Beremend 26 (Hungary) (Kessler 2010); Q1: Betfia 9 (Gál 2002).

- Sylvia sp.

From sites in Europe outside the Carpathian Basin Q3: France, Italy; Q4: Bosnia-Herczegovina, Greece, United Kingdom (Tyrberg 1998).

- Locustella Kaup, 1829

- Locustella $\dagger$ kordosi Kessler, 2013

Type locality and age: Polgárdi 5, Upper Miocene (MN 13) (Hungary) (Kessler 2013a, 2013b). Corresponds in characteristics to species of extant genus.

\section{- Locustella $\uparrow$ janossyi Kessler, 2013}

Type locality and age: Csarnóta 2, Pliocene (MN 15) (Hungary) (Kessler 2013a, 2013b). Other locality and age: Beremend 26, Pliocene (MN 15) (Hungary) (Kessler 2013a, 2013b). Corresponds in its characteristics to extant genus and in dimensions with smaller-sized extant species.

- Locustella † magna Kessler, 2013

Type locality and age: Beremend 26, Pliocene (MN 15) (Hungary) (Kessler 2013a, 2013b). Corresponds in its characteristics to extant species, but it is larger.

- Locustella fluviatilis (Wolf, 1810)

Q1: Betfia 9 (Romania) (Gál 2002).

- Locustella sp. foss. indet.

MN 9: Rudabánya (Hungary) (Kretzoi 1975, Jánossy 1993).

Outside of the Carpathian Basin the genus is known only from the Late Pleistocene (Q4) of the Czech Republic as Locustella naevia (Boddaert, 1783) (Tyrberg 1998). 


\section{- Phylloscopus † miocaenicus Kessler et Hír, 2012}

Type locality and age: Felsőtárkány, Middle Miocene (MN 7-8) (Hungary) (Kessler \& Hír 2012).

By its characteristics, it belongs to the genus Phylloscopus of the Sylviidae family.

- Phylloscopus $\dagger$ venczeli Kessler, 2013

Type locality and age: Polgárdi 5, Upper Miocene (MN 13) (Hungary) (Kessler 2013a, 2013b). Corresponds in its characteristics to extant species of the genus.

\section{- Phylloscopus $\uparrow$ pliocaenicus Kessler, 2013}

Type locality and age: Csarnóta 2, Pliocene (MN 15) (Hungary) (Kessler 2013a, 2013b). Its characteristic corresponds to the extant genus.

\section{- Phylloscopus sp. indet.}

Q3/I: Hundsheim (Austria) (Lambrecht 1933, Jánossy 1974, 1979).

Outside of Carpathian Basin the genus is known the Late Pliocene from Varsets (MN 17, Bulgaria) as Phylloscopus sp. (Boev 1996, 2000); from Cerdzenica - Bulgaria (Lower Pleistocene, Q1) (Boev 2000) and in the Late Pleistocene (Q4) with extant species (Phylloscopus bonelli, P. collybita, P. trochilus, P. sibilatrix) from Czech Republic, France, Italy, Switzerland (Tyrberg 1998).

- Regulus Vieillot, 1807

- Regulus $\uparrow$ plioceanicus Kessler, 2013

Type locality and age: Beremend 26, Pliocene (MN 15) (Hungary) (Kessler 2013a, 2013b). Corresponds in its characteristics to extant species, but it is larger in its sizes.

\section{- Regulus sp.}

Q3/I: Hundsheim (Austria) (Lambrecht 1933, Jánossy 1974, 1979); Q4/II: RépáshutaRejteki Niche (Hungary) (Jánossy 1962, 1979, 1986).

The genus is known from the extinct species Regulus bulgaricus Boev, 1999 from Varshets - Bulgaria (Late Pliocene, MN 17) (Boev 1999). The extant species was reported from S'Onix - Mallorca, Spain (Early Pleistocene, Q1) (Sondaar et al. 1995) and as Regulus regulus and Regulus ignicapilus from Czech Republic, Poland Spain and Switzerland of the Late Pleistocene from Europa (Tyrberg 1998).

- Sylviidae gen. et sp. foss. indet.

MN 6: Kőalja 2 (Subpiatra) (Romania) (Kessler \& Venczel 2009).

The family is identified from the Miocene and Pliocene only in the Carpathian Basin and in Bulgaria.

\section{Fam. Motacillidae Vigors, 1825}

- Anthus Bechstein, 1807

- Anthus $\uparrow$ antecedens Kessler et Hír, 2012

Type locality and age: Felsőtárkány, Middle Miocene (MN 7-8) (Hungary) (Kessler \& Hír 2012). It is assigned to pipits with larger stature.

\section{- Anthus † hiri Kessler, 2013}

Type locality and age: Polgárdi 5, Upper Miocene (MN 13) (Hungary) (Kessler 2013a, 2013b). It corresponds in its characteristics to the extant genus Anthus, its sizes are between extant $A$. spinoletta and $A$. trivialis, it belongs to the pipits with a smaller stature. 


\section{- Anthus $\dagger$ baranensis Kessler, 2013}

Type locality and age: Csarnóta 2, Pliocene (MN 15) (Hungary) (Kessler 2013a, 2013b). It corresponds in its characteristics to smaller-size extant species.

- Anthus pratensis (Linnaeus, 1758)

Q4/I: Krapina (Croatia) (V. Malez 1973, 1984, V. Malez-Bačić 1975), Hámor-Puskaporos Niche (Hungary) (Lambrecht 1912, 1916, 1933, Jánossy 1979, 1986). From sites in Europe outside the Carpathian Basin Q3: France; Q4: Austria, Bosnia-Herczegovina, Croatia, Czech Republic, France, Ireland, Spain, United Kingdom (Tyrberg 1998).

- Anthus cervinus (Pallas, 1811)

Q3/I: Hundsheim (Austria) (Jánossy 1974).

- Anthus trivialis (Linnaeus, 1758)

Q1: Betfia 9 (Romania) (Gál 2002); Q4/I: Velika Pecina (Croatia) (V. Malez 1975, 1984, 1988); Hámor-Puskaporos Niche (Hungary) (Lambrecht 1912, 1916, 1933, Jánossy 1979, 1986), Hidegszamos-Csont Cave (Someşul Rece, Peştera cu Oase) (Romania) (Lambrecht 1915); Q4/II: Herkulesfürdő-Rablók Cave (Băile Herculane, Peştera Hoţilor) (Romania) (Kessler 1981, Gál 2002). From sites in Europe outside the Carpathian Basin Q3: Czech Republic, France; Q4: Bulgaria, Croatia, France, Georgia, Italy, Poland, Ukraine, United Kingdom (Tyrberg 1998).

\section{- Anthus spinoletta (Linnaeus, 1758)}

Q2: Kövesvárad (Hungary) (Jánossy 1963); Q4/I: Óruzsin-Antal Cave (Oruzer, Antal Cave, Slovakia) (Nehring 1880, Róth 1881, Lambrecht 1912, 1933). From sites in Europe outside the Carpathian Basin; Q3: France, Germany, Spain; Q4: Austrias, France, Spain, Switzerland, United Kingdom (Tyrberg 1998).

- Anthus sp. foss. indet.

MN 16: Beremend 15 (Hungary) (Jánossy 1992); Q1: Betfia 2 (Kormos 1913, Čapek 1917, Lambrecht 1933, Kessler 1975, Jánossy 1979, Gál 2002), Betfia 9 (Kessler 1975, Gál 2002) (all in Romania).

\section{- Anthus sp.}

Q4/I: Mixnitz-Drachenhöhle (Austria) (Lambrecht 1933); Pilisszántói I. Niche (Hungary) (Lambrecht 1915, 1933, Jánossy 1979, 1986). From sites in Europe outside the Carpathian Basin; Q3: Czech Republic, Georgia, Italy; Q4: Belgium, Georgia, Switzerland, Ukraine (Tyrberg 1998).

On outside the Carpathian Basin the genus is known from Rebielice Królowskie 1 - Poland (Upper Pliocene MN 16) (Jánossy 1974); Varseths - Bulgaria (Upper Pliocene, MN 16, MN 17) (Boev 1996, 2000). The fossil species Anthus bosniaskii Pycraft 1909 from Gabbro - Italy (Upper Miocene, MN 13) was put by Mlíkovský into „Family incertae sedis” (Mlíkovský 2002).

\section{- Motacilla Linnaeus, 1758}

\section{- Motacilla $\uparrow$ intermedia Kessler, 2013}

Type locality and age: Polgárdi 5, Upper Miocene (MN 13) (Hungary) (Kessler 2013a, 2013b). The sizes are intermediate between $M$. alba and $M$. cinerea, but in morphological characteristics it resembles M. alba. In several characteristics, it exhibits the mixture of Anthus and Motacilla types. The remains were reported as Motacilla sp. by Jánossy $(1991,1995)$. 


\section{- Motacilla † minor Kessler, 2013}

Type locality and age: Beremend 26, Pliocene (MN 15) (Hungary) (Kessler 2013a, 2013b). It corresponds in its characteristics with extant Motacilla flava but has intermediate dimensions between M. flava and M. cinerea.

- Motacilla $\dagger$ robusta Kessler, 2013

Type locality and age: Beremend 26, Pliocene (MN 15) (Hungary) (Kessler 2013a, 2013b). It is more robust than the extant species.

\section{- Motacilla flava Linnaeus, 1758}

Q1: Betfia 9 (Romania) (Gál 2002); Q4/I: Körösmart (Rîpa) (Romania) (Jánossy in Hamar \& Csák 1969, Kessler 1974, Gál 2002). From sites in Europe outside the Carpathian Basin Q3: France; Q4: Croatia, France, Switzerland (Tyrberg 1998).

\section{- Motacilla alba Linnaeus, 1758}

Q1: Betfia 2 (Kormos 1913, Čapek 1917, Lambrecht 1933, Kessler 1975, Jánossy 1979, Gál 2002), Betfia 9 (Kessler 1975, Gál 2002) (all in Romania); Q4/I: Pilisszántói I. Niche (Hungary) (Lambrecht 1915, 1933, Jánossy 1979, 1986); Q4/II: Körösbánlaki Cave (Peştera din Bălnaca), Kőrösmart (Rîpa) (Kessler 1982), Révi Caves (Peşterile din Vadu Crișului), Szegyestel-Drăcoaia Cave (Sighiştel, Peştera Drăcoaia) (Kessler 1982) (all in Romania). From sites in Europe outside the Carpathian Basin Q3: Czech Republic, France, Russia; Q4: Austria, Czech Republic, Croatia, France, Germany, Poland, Russia, Ukraine, United Kingdom (Tyrberg 1998).

\section{- Motacilla cinerea Tunstall, 1771}

Q1: Betfia 9 (Romania) (Gál 2002); Q4/II: Szegyestel-völgyi Caves (peşteri din Valea Sighiştelului) (Romania) (Kessler 1982). From sites in Europe outside the Carpathian Basin Q4: Croatia, United Kingdom (Tyrberg 1998).

\section{- Motacilla sp. foss. indet.}

MN 7-8: Mátraszőlős 1 (Hungary) (Kessler \& Hír 2012);

The fossil species from Polgárdi and Beremend 26 were reported previously as Motacilla sp. foss. indet. by Jánossy $(1991,1995)$ and Kessler $(2010)$.

\section{- Motacilla sp.}

Q3/I: Hundsheim (Austria) (Lambrecht 1933, Jánossy 1979, Mlikovskỳ 1998, 2002). From sites in Europe outside the Carpathian Basin Q3: Czech Republic; Q4: France, Germany, United Kingdom (Tyrberg 1998).

The genus was described outside of the Carpathian Basin from Varshets - Bulgaria (Upper Pliocene, MN 17) by Boev (1996, 2000), and from Stránská skálá - Czech Republic (Lower Pleistocene, MQ1) by Mlíkovský (1995). The fossil species Motacilla humata Milne-Edwards 1871 and Motacilla major Milne-Edwards 1871 (Milne-Edwards 1871) from Saint-Gerand-lePuy - France (Lower Miocene, MN 2) has a disputed situation (Mlíkovský 2002).

\section{Fam. Bombycillidae Swainson, 1832}

- Bombycilla Swainson, 1832

\section{- Bombycilla † hamori Kessler et Hír, 2012}

Type locality and age: Litke 2, Lower Miocene (MN 5) (Hungary) (Kessler \& Hír 2012). Other locality and age: Felsőtárkány, Middle Miocene (MN 7-8) (Hungary) (Kessler \& Hír 
2012). Based on morphological characteristics, it is a fossil species with smaller size than the extant Bombycilla garrulus.

- Bombycilla $\uparrow$ brevia Kessler, 2013

Type locality and age: Polgárdi 5, Upper Miocene (MN 13) (Hungary) (Kessler 2013a, 2013b).

The dimensions are much smaller than in the extant species.

- Bombycilla $\uparrow$ kubinyii Kessler, 2013

Type locality and age: Beremend 26, Pliocene (MN 15) (Hungary) (Kessler 2013a, 2013b). It corresponds in its characteristics and size to extant species. This species was reported initially as Bombycilla sp. foss. indet. by Kessler (2010).

- Bombycilla garrulus (Linnaeus, 1758)

Q3/I: Vindija (Croatia) (M. Malez 1961, V. Malez 1973, 1988); Q4/I: Velika Pecina (Croatia) (V. Malez 1975, 1984, 1988); Szilvásvárad-Istállóskői Cave (Hungary) (Lambrecht 1912, 1933, Jánossy 1952, 1955, 1979, 1986); Szegyestel-Măgura Cave (Sighiştel, Peştera Măgura) (Romania) (Kessler 1982, 1985, Gál 2002).From sites in Europe outside the Carpathian Basin Q3: France; Q4: Belgium, Bosnia-Herczegovina, Croatia, Czech Republic, France, Italy, Poland, United Kingdom (Tyrberg 1998).

- Bombycilla sp. foss. indet.

MN 15: Csarnóta 2 (Hungary) (Kessler 2010a); Q1: Beremend 17 (Hungary) (Jánossy 1992); Betfia 9 (Romania) (Gál 2002).

The family and genus are known from fossil species in the Neogene only from the Carpathian Basin.

\section{Fam. Troglodytidae Vieillot, 1807}

- Troglodytes Vieillot, 1807

- Troglodytes $\uparrow$ robustus Kessler, 2013

Type locality and age: Polgárdi 5, Upper Miocene (MN 13) (Hungary) (Kessler 2013a, 2013b). The fossil species differs to the extant in its larger size.

- Troglodytes troglodytes (Linnaeus, 1758)

Q4/I: Velika Pecina (Croatia) (V. Malez 1975, 1984, 1988).

The genus is known to extant species from S'Onix - Mallorca - Spain (Early Pleistocene, MN 18) (Sondaar et al. 1995) and from Q3: France; Q4: Croatia, France, Poland, United Kingdom (Tyrberg 1998).

\section{Fam. Cinclidae Cabanis, 1847}

- Cinclus Borkhausen, 1897

- Cinclus $\uparrow$ major Kessler et Hír, 2012

Type locality and age: Litke 2, Lower Miocene (MN 5) (Hungary) (Kessler \& Hír 2012). The features of the bone are similar to those of the extant species, but their dimensions are slightly larger.

\section{- Cinclus $\uparrow$ gaspariki Kessler, 2013}

Type locality and age: Polgárdi 5, Upper Miocene (MN 13) (Hungary) (Kessler 2013a, 2013b). The remains in general correspond in characteristics to the extant species. 


\section{- Cinclus $\uparrow$ minor Kessler, 2013}

Type locality and age: Csarnóta 2, Middle Pliocene (MN 15-16) (Hungary) (Kessler 2013a, 2013b). It is smaller than the extant species.

\section{- Cinclus cinclus (Linnaeus, 1758)}

Q1: Betfia 9 (Romania) (Gál 2002); Q4/I: Merkenstein (Austria) (Wettstein \& Mühlhofer 1938), Hámor-Herman Ottó Cave (Lambrecht 1915, 1933), Pilisszántói I. Niche (Lambrecht 1915, 1933, Jánossy 1979, 1986) (all in Hungary); Barcarozsnyó-Gura Cheii Cave (Peștera Gura Cheii-Râşnov) (Romania) (Gál 1998, 2002); Q4/II: Grosse Offenbergerhöhle (Austria) (Bocheński \& Tomek 1994); Peterd-Tordai-Defile-Magyar Cave (Petrești, Cheile Turzii-Peștera Ungurească) (Kessler \& Gál 1998, Gál 2005a), Vársonkolyos-Izbindis Cave (Şuncuiuş, Peştera Izbîndiş) (Kessler 1977, Gál 2002) (all in Romania).

It is also known in the Middle Pleistocene (Q3) from localities in France and Germany, and in the Late Pleistocene (Q4) in Austria, Belgium, Czech Republic, France, Germany, Italy, United Kingdom (Tyrberg 1998).

\section{Fam. Prunellidae Richmond, 1908}

- Prunella Vieillot, 1818

- Prunella $\dagger$ freudenthali Kessler, 2013

Type locality and age: Polgárdi 5, Upper Miocene (MN 13) (Hungary) (Kessler 2013a, 2013b). The sizes of the humerus corresponds to the extant species P. modularis. The sizes of ulna and femur is slightly smaller than in the extant species.

\section{- Prunella $\uparrow$ kormosi Kessler, 2013}

Type locality and age: Csarnóta 2, Pliocene (MN 15) (Hungary) (Kessler 2013a). Other locality and age: Beremend 26, Pliocene (MN 15) (Hungary) (Kessler 2013a). It is larger than the extant $P$. modularis but smaller than P. collaris.

\section{- Prunella modularis (Linnaeus, 1758)}

Q4/I: Grosse Badlhöhle (Austria) (Fladerer 1993); Esküllö-Igric Cave (Aștileu, Peştera Igriţa) (Romania) (Kessler 1985). From sites in Europe outside the Carpathian Basin Q12: Spain; Q3: France, Italy; Q4: Austria, Germany, Spain, Ukraine, United Kingdom (Tyrberg 1998).

\section{- Prunella collaris (Scopoli, 1769)}

Q4/II: Herkulesfürdö-Zoltán Cave (Băile Herculane, Peştera Zoltan) (Gál 2002), Kazánszoros-Töröklik Cave (Cazanele Mari, Peştera Cuina Turcului) (Kessler 1974, Fischer \& Stephan 1977) (all in Romania). From sites in Europe outside the Carpathian Basin Q3: Czech Republic, France, Greece, Spain; Q4: Austria, France, Germany, Greece, Italy, Spain, Switzerland (Tyrberg 1998).

The genus is not known outside the Carpathian Basin with fossil species.

\section{Fam. Laniidae Swainson, 1834}

- Lanius Linnaeus, 1758

\section{- Lanius † schreteri Kessler et Hír, 2012}

Type locality and age: Felsőtárkány, Middle Miocene (MN 7-8) (Hungary) (Kessler \& Hír 2012). 
Other locality: Felnémet 2/3, Middle Miocene (MN 7-8) (Hungary) (Kessler \& Hír 2012). Based on its characteristics, it is equivalent to Lanius excubitor, though larger than it.

\section{- Lanius † capeki Kessler, 2013}

Type locality and age: Polgárdi 5, Upper Miocene (MN 13) (Hungary) (Kessler 2013a, 2013b). In general, it corresponds in characteristics and sizes to the extant $L$. collurio.

\section{- Lanius $\dagger$ hungaricus Kessler, 2013}

Type locality and age: Csarnóta 2, Pliocene (MN 15) (Hungary) (Kessler 2013a, 2013b). It corresponds in size to extant Lanius collurio.

\section{- Lanius † major Kessler, 2013}

Type locality and age: Beremend 26, Pliocene (MN 15) (Hungary) (Kessler 2013a, 2013b). The remains derive from two different-sized specimens. The humerus is mostly smaller than the extant L. excubitor, the carpometacarpus and tarsometatarsus derived from the large specimens.

\section{- Lanius † intermedius Kessler, 2013}

Type locality and age: Beremend 26, Pliocene (MN 15) (Hungary) (Kessler 2013a, 2013b).

It has intermediate dimensions between extant species L. minor and L. collurio, and differs in characteristics to the much smaller L. hungaricus Kessler, 2012 from Csarnóta.

\section{- Lanius excubitor Linnaeus, 1758}

Q1: Betfia 9 (Romania) (Gál 2002); Q3/II: Vindija (Croatia) (M. Malez 1961, V. Malez 1973, 1988); Q4/I: Szegyestel-Măgura Cave (Sighiştel, Peştera Măgura) (Romania) (Kessler 1982, 1985, Gál 2002); Q4/II: Kevélynyergi Cave (Hungary) (Kessler 2010); Szegyestel-Drăcoaia Cave (Sighiştel, Peştera Drăcoaia) (Romania) (Kessler 1982). From sites in Europe outside the Carpathian Basin Q4: Austria, Croatia, France, Germany, Poland, Spain, Switzerland (Tyrberg 1998).

\section{- Lanius collurio Linnaeus, 1758}

Q2: Betfia 7 (Romania) (Kessler 1975, Gál 2002); Q4/I: Merkenstein (Austria) (Wettstein \& Mühlhofer 1938); Budapest-Remetehegyi Niche (Hungary) (Kormos 1914, Lambrecht 1933, Jánossy 1979, 1986). From sites in Europe outside the Carpathian Basin Q4: Austria, Bulgaria, Czech Republic, France, Germany, Moldova, Ukraine, United Kingdom (Tyrberg 1998).

\section{- Lanius minor Gmelin, 1788}

Q1: Betfia 2 (Kormos 1913, Čapek 1917, Lambrecht 1933, Kessler 1975, Jánossy 1979, Gál 2002), Betfia 9 (Kessler 1975, Gál 2002) (all in Romania); Q4/I: Pilisszántói I. Niche (Hungary) (Lambrecht 1915, 1933, Jánossy 1979, 1986); Szegyestel-Măgura Cave (Sighiştel, Peştera Măgura) (Romania) (Kessler 1982, 1985, Gál 2002); Q4/II: Révi Caves (Peşterile din Vadu Crișului) (Romania) (Kessler 1982). From sites in Europe outside the Carpathian Basin Q1-2: Greece; Q4: Spain (Tyrberg 1998).

\section{- Lanius senator Linnaeus, 1758}

Q4: Pilisszántó 1 (Lambrecht 1915, 1933, Jánossy 1986), Puskaporos (Lambrecht 1933, Jánossy 1986) (all in Hungary). From sites in Europe outside the Carpathian Basin Q3: France, Italy, Spain; Q4: Austria, France, Spain (Tyrberg 1998).

\section{- Lanius sp.}

From sites in Europe outside the Carpathian Basin Q4: Austria, Bosnia-Herczegovina, Spain (Tyrberg 1998). 


\section{- Lanius sp. foss. indet.}

MN 16: Betfia 13 (Romania) (Kessler 1975, Gál 2002 - as Lanius collurio);

The fossil species from Beremend 26 were reported previously as Lanius sp. foss. indet. by Kessler (2010).

- Laniidae gen. et sp. foss. indet.

MN 6: Köalja 2 (Subpiatra) (Romania) (Kessler \& Venczel 2009).

The family and genus are known outside the Carpathian Basin from the Late Pliocene from of Varshets (MN 17, Bulgaria) as Lanius sp. (Boev 1996, 2000); from Petralona 24 Greece (Lower Pleistocene) as Lanius cf. minor Gmellin, 1788 by Kretzoi (1977). The fossil species Lanius miocaenus Milne-Edwards, 1871 (Milne-Edwards 1869-71) from SaintGérand-le-Puy - France (Lower Miocene, MN 2) was put into "Family incertae sedis" by Mlíkovský (2002).

\section{Fam. Sturnidae Vigors, 1825}

- Sturnus Linnaeus, 1758

- Sturnus $\dagger$ kretzoii Kessler et Hír, 2012

Type locality and age: Rudabánya, Upper Miocene (MN 9) (Hungary) (Kessler \& Hír 2012). The features of the remains correspond to the extant genus, but their dimensions are much smaller.

\section{- Sturnus $\uparrow$ brevis Kessler, 2013}

Type locality and age: Polgárdi 5, Upper Miocene (MN 13) (Hungary) (Kessler 2013a, 2013b). It differs in its smaller sizes from the extant species.

\section{- Sturnus $\uparrow$ pliocaenicus Kessler, 2013}

Type locality and age: Beremend 26, Pliocene (MN 15) (Hungary) (Kessler 2013a, 2013b). It differs to known extinct and extant species with much larger dimensions.

\section{- Sturnus $\dagger$ baranensis Kessler, 2013}

Type locality and age: Beremend 26, Middle Pliocene (MN 15-16) (Hungary) (Kessler 2013a, 2013b). It differs from extant species in its intermediate dimensions between S. vulgaris and $S$. roseus.

\section{- Sturnus vulgaris Linnaeus, 1758}

Q1: Betfia 2 (Kormos 1913, Čapek 1917, Lambrecht 1933, Kessler 1975, Jánossy 1979, Gál 2002), Betfia 9 (Kessler 1975, Gál 2002) (all in Romania); Q2: Betfia 5 (Romania) (Kessler 1975, Jánossy 1979, Gál 2002); Q3/II: Vindija (Croatia) (M. Malez 1961, V. Malez 1973, 1988, M. Malez \& Rukavina 1979); Q4/I: Bajót, Herman Ottó Cave, Csobánka-Kiskevélyi Cave (Lambrecht 1912, 1915, 1933, Jánossy 1979), Hámor-Puskaporos Niche (Lambrecht 1912, 1916, 1933, Jánossy 1979, 1986), Hámor-Herman Ottó Cave (Lambrecht 1915, 1933), Pilisszántói I. Niche (Lambrecht 1915, 1933, Jánossy 1979, 1986), TatabányaKálváriahegy 4. Cave (Gál 2005a, 2005b), Varbó-Lambrecht Kálmán Cave (Jánossy 1964, 1979) (all in Hungary); Nándor-Nándori Cave (Nandru-Peştera Curata) (Romania) (Jánossy 1965, Fischer \& Stephan 1977, Kessler 1985, Jurcsák \& Kessler 1988, Gál 2002, 2003); Q4/II: Ecsegfalva (Pike-Tay et al. 2004, Gál 2007), Szendrő (Gál 2005b, Tassi 2006) (all in Hungary); Herkulesfürdö-Rablók Cave (Băile Herculane, Peştera Hoţilor) (Kessler 1981, Gál 2002), Kazánszoros-Töröklik Cave (Cazanele Mari, Peştera Cuina Turcului) (Kessler 
1974, Fischer \& Stephan 1977), Körösbánlaki Cave (Peştera din Bălnaca) (Kessler 1982), Révi Caves (Peşterile din Vadu Crișului) (Kessler 1982) (all in Romania). From sites in Europe outside the Carpathian Basin Q3: Czech Republic France, Malta, United Kingdom; Q4: Austria, Bosnia-Herzegovina, Croatia, France, Germany, Greece, Italy, Poland, Eussia, Spain, Switzerland, Ukarine, United Kingdom (Tyrberg 1998).

\section{- Sturnus roseus (Linnaeus, 1758)}

Q4/I: Pilisszántói I. Niche (Hungary) (Lambrecht 1915, 1933, Jánossy 1979, 1986). From sites in Europe outside the Carpathian Basin Q4: France, Italy (Tyrberg 1998).

\section{- Sturnus unicolor Temminck, 1820}

From sites in Europe outside the Carpathian Basin Q4: France, Spain (Tyrberg 1998).

\section{- Sturnus sp.}

Q1: Beremend 16, 17 (Hungary) (Jánossy 1992); Q4/I: Szilvásvárad-Istállóskői Cave (Hungary) (Lambrecht 1912, 1933, Jánossy 1952, 1955, 1979, 1986). From sites in Europe outside the Carpathian Basin Q3: Italy, Spain, Turkey; Q4: France, Germany, Greece, Italy, Spain, United Kingdom (Tyrberg 1998).

The family and genus were described outside the Carpathian Basin as Sturnus sp. in the Late Pliocene and the Early Pleistocene localities from Varseths - Bulgaria (MN 17 - MQ1) by Boev (1996, 2000), West Runton and Boxgrove - England (Harrison 1979, Harrison \& Stewart 1999) and Prezletice - Czech Republic (Čapek 1917, Jánossy 1983, 1992).

\section{Fam. Passeridae Illiger, 1811}

\section{- Passer Koch, 1816}

\section{- Passer † hiri Kessler, 2013}

Type locality and age: Polgárdi 5, Upper Miocene (MN 13) (Hungary) (Kessler 2013a, 2013b). Its size is smaller than the extant species but corresponds to it in its characteristics.

\section{- Passer $\uparrow$ minusculus Kessler, 2013}

Type locality and age: Csarnóta 2, Pliocene (MN 15) (Hungary) (Kessler 2013a, 2013b). Is very little in comparison to extant species of the genus but corresponds it in its characteristics.

\section{- Passer † pannonicus Kessler, 2013}

Type locality and age: Beremend 26, Pliocene (MN 15) (Hungary) (Kessler 2013a, 2013b). It corresponds mostly in dimensions and characteristics to extant species. The extinct species from Polgárdi and Csarnóta are smaller.

\section{- Passer montanus (Linnaeus, 1758)}

Q1: Betfia 2 (Romania) (Kormos 1913, Čapek 1917, Lambrecht 1933, Kessler 1975, Jánossy 1979, Gál 2002); Q2: Betfia 5 (Romania) (Kessler 1975, Jánossy 1979, Gál 2002); Q3: Uppony I/1 (Hungary) (Jánossy 1979); Q4/I: Velika Pecina (Croatia) (V. Malez 1975, 1984, 1988); Q4/II: Szegyestel-Drăcoaia Cave (Sighiştel, Peştera Drăcoaia) (Romania) (Kessler 1982). From sites in Europe outside the Carpathian Basin Q3: France; Q4: Bulgaria, France, Italy, Ukraine, United Kingdom (Tyrberg 1998).

\section{- Passer domesticus (Linnaeus, 1758)}

Q4/I: Hámor-Puskaporos (Hungary) (Lambrecht 1912, 1916, 1933, Jánossy 1979, 1986); Q4/II: Ecsegfalva (Pike-Tay et al. 2004, Gál 2007), Legény Cave (Lambrecht 1914) (all in 
Hungary). From sites in Europe outside the Carpathian Basin Q3: France; Q4: Czech Republic, France, Ireland, Spain, Ukraine, United Kingdom (Tyrberg 1998).

- Passer sp.

From sites in Europe outside the Carpathian Basin Q3: Ukraine; Q4: Bosnia-Herczegovina, Germany, Italy, Spain (Tyrberg 1998).

The earliest report of the family and the genus is from Saint-Gérand-le-Puy - France (Lower Miocene, MN 2) as Passer sp. (Mourer-Chauviré 1995), but it is not known in other localities from Neogene.

\section{Fam. Fringillidae LeACH, 1820}

\section{- Serinus Koch, 1916}

- Serinus serinus (Linnaeus, 1766)

Q1: Németóvár 4B (Deutsch-Altenburg) (Austria) (Jánossy 1981). From sites in Europe outside the Carpathian Basin Q3: France, United Kingdom (Tyrberg 1998).

\section{- Serinus citrinella (Pallas, 1764)}

From sites in Europe outside the Carpathian Basin Q3: France; Q4: France, Poland (Tyrberg 1998).

\section{- Serinus sp.}

From sites in Europe outside the Carpathian Basin Q3: Italy; Q4: France (Tyrberg 1998).

- Serinus sp. foss. indet.

MN 16: Beremend 15 (Hungary) (Jánossy 1992, 1996);

The genus Serinus sp. was reported from Saint-Gerand-le Puy - France (Lower Miocene, MN 2), (Mourer-Chauviré 1995).

\section{- Carduelis Brisson, 1760}

- Carduelis $\uparrow$ kretzoii Kessler, 2013

Type locality and age: Polgárdi 5, Upper Miocene (MN 13) (Hungary) (Kessler 2013a, 2013b). It corresponds in its sizes to extant smaller and medium size extant species of the genus, such as $C$. carduelis, C. flammea and C. spinus.

\section{- Carduelis † lambrechti Kessler, 2013}

Type locality and age: Polgárdi 5, Upper Miocene (MN 13) (Hungary) (Kessler 2013a, 2013b). Its size corresponds to extant Carduelis chloris.

\section{- Carduelis † parvulus Kessler, 2013}

Type locality and age: Csarnóta 2, Pliocene (MN 15) (Hungary) (Kessler 2013a, 2013b). It corresponds in characteristics to genus and in dimensions to little sized species.

\section{- Carduelis $\uparrow$ medius Kessler, 2013}

Type locality and age: Csarnóta 2, Pliocene (MN 15) (Hungary) (Kessler 2013a, 2013b). Corresponds in its characteristics and sizes with one medium sized extant species of the genus.

- Carduelis chloris Linnaeus, (1758)

Q1: Betfia 9 (Romania) (Gál 2002); Q4/I: Merkenstein (Austria) (Wettstein \& Mühlhofer 1938); Ohábaponor-Bordu Mare Cave (Ohaba Ponor-Peştera Bordu Mare) (Kessler 1985, Jurcsák \& Kessler 1988, Gál 2002, 2003), Szegyeste-Măgura Cave (Sighiştel, Peştera Măgura) (Kessler 1982, 1985, Gál 2002) (all in Romania); Q4/II: Révi Caves (Peşterile din Vadu Crişului), Szegyestel-Drăcoaia Cave (Sighiştel, Peştera Drăcoaia), Szegyestel-völgyi Caves 
(peşteri din Valea Sighiştelului) (Kessler 1982) (all in Romania). From sites in Europe outside the Carpathian Basin Q1-2: France; Q3: France, Italy, Malta; Q4: Bosnia-Hercezgovina, France, Germany, Greece, Ireland, Italy, Poland, Russia, Spain, Ukraine, United Kingdom (Tyrberg 1998).

- Carduelis carduelis (Linnaeus, 1758)

Q1: Betfia 9 (Romania) (Gál 2002); Q4/I: Velika Pecina (Croatia) (V. Malez 1975, 1984, 1988). From sites in Europe outside the Carpathian Basin Q1-2: Spain; Q3: Czech Republic, France; Q4: France, Germany, Italy, Poland, Russia, Ukraine, United Kingdom (Tyrberg 1998).

\section{- Carduelis spinus (Linnaeus, 1758)}

Q1: Betfia 9 (Romania) (Gál 2002). From sites in Europe outside the Carpathian Basin Q3: Czech Republic; Q4: France, Germany, United Kingdom (Tyrberg 1998). From sites in Europe outside the Carpathian Basin Q1-2: France; Q4: Czech Republic, Russia (Tyrberg 1998).

\section{- Carduelis cannabina (Linnaeus, 1758)}

Q1: Betfia 9 (Romania) (Gál 2002); Q4/I: Körösmart (Rîpa) (Jánossy in Hamar \& Csák 1969, Kessler 1974a, Gál 2002); Szegyestel-Măgura Cave (Sighiştel, Peştera Măgura) (Kessler 1982, 1985, Gál 2002) (all in Romania). From sites in Europe outside the Carpathian Basin Q3: France, Ukraine; Q4: Austria, Czech Republic, France, Germany, Ireland, Italy, Poland, Spain, United Kingdom (Tyrberg 1998).

\section{- Carduelis flammea (Linnaeus, 1758)}

Q4/I: Merkenstein (Austria) (Wettstein \& Mühlhofer 1938). From sites in Europe outside the Carpathian Basin Q3: Czech Republic; Q4: France, Germany, United Kingdom (Tyrberg 1998). From sites in Europe outside the Carpathian Basin Q3: France; Q4: Austria, France, Italy, Spain, United Kingdom (Tyrberg 1998).

\section{- Carduelis sp. indet.}

Q1: Beremend 17 (Hungary) (Jánossy 1992); Betfia 9 (Romania) (Gál 2002); Q4/I: Tatabánya-Kálvária-hegy 4. Cave (Hungary) (Gál 2005a, 2005b). From sites in Europe outside the Carpathian Basin Q1-2: France; Q3: Italy; Q4: Belgium, Czech Republic, Russia (Tyrberg 1998).

The genus was described outside of the Carpathian Basin from the Late Pliocene Early Pleistocene, (MN 17-MQ1) in Varshets and Cerzenica. Bulgaria by Boev (1996, 2000), Quibas and S'Onix-Spain by Montoya et al. (1999) and Sondaar et al. (1995); Mas Ramboult - France by Mourer-Chauviré (1995) and Stránská skála - Czech Republic by Jánossy (1972).

\section{- Pinicola Vieillot, 1807}

\section{- Pinicola $\uparrow$ kubinyii Kessler, 2013}

Type locality and age: Csarnóta 2, Pliocene (MN 15) (Hungary) (Kessler 2013a, 2013b). It is smaller in dimensions than the extants species.

\section{- Pinicola enucleator (Linnaeus, 1758)}

Q1: Németóvár 4B (Deutsch-Altenburg) (Austria) (Jánossy 1981); Q4/I: Merkenstein (Austria) (Wettstein \& Mühlhofer 1938); Pilisszántói I. Niche (Lambrecht 1915, 1933, Jánossy 1979, 1986), Szilvásvárad-Istállóskői Cave (Lambrecht 1912, 1933, Jánossy 1952, 
1955, 1979, 1986) (all in Hungary); Q4/II: Herkulesfürdö-Zoltán Cave (Băile Herculane, Peştera Zoltan) (Romania) (Gál 2002). From sites in Europe outside the Carpathian Basin Q3: France; Q4: France, Italy, Spain, United Kingdom (Tyrberg 1998).

\section{- Pinicola sp.}

Q3/I: Hundsheim (Austria) (Lambrecht 1933, Jánossy 1974a, 1979); Tarkő (Kessler 2010), Vértesszőlős 2 (Jánossy 1974a, 1979) (all in Hungary).

- Coccothraustes Brisson, 1760

- Coccothraustes $\uparrow$ major Kessler, 2013

Type locality and age: Beremend 26, Pliocene (MN 15) (Hungary) (Kessler 2013a, 2013b). It differs from extant species in its dimensions and larger size and corresponds much in its characteristics. It should be noted that we do not come across this genus in other songbird-rich materials (Polgárdi, Csarnóta).

\section{- Coccothraustes coccothraustes (Linnaeus, 1758)}

Q1: Betfia 2 (Kormos 1913, Čapek 1917, Lambrecht 1933, Kessler 1975, Jánossy 1979, Gál 2002), Betfia 9 (Kessler 1975, Gál 2002) (all in Romania); Q3/I: Hundsheim (Austria) (Mlíkovskỳ 2009); Q3/II: Süttő 1-4 (Hungary) (Jánossy 1979); Q4/I: Merkenstein (Wettstein \& Mühlhofer 1938), Velika Pecina (V. Malez 1975, 1984, 1988), Velika pec na Lipi (V. Malez 1975, 1984, 1993, V. Malez-Bacic 1975, 1979) (all in Croatia); Hámor-Herman Ottó Cave (Lambrecht 1915, 1933), Szilvásvárad-Istállóskői Cave (Labrecht 1912, 1933, Jánossy 1952, 1955, 1979, 1986) (all in Hungary); Ohábaponor-Bordu Mare Cave (Ohaba Ponor-Peştera Bordu Mare) (Kessler 1985, Jurcsák \& Kessler 1988, Gál 2002, 2003), Szegyestel-Măgura Cave (Sighiştel, Peştera Măgura) (Kessler 1982, 1985, Gál 2002) (all in Romania); Óruzsin-Antal Cave (Oruzer, Antal Cave) (Slovakia) (Nehring 1880, Róth 1881, Lambrecht 1933); Q4/II: Körösbánlaki Cave (Peştera din Bălnaca) (Kessler 1982), Remetelórév-Bólyiköi Cave (Lorău-Peştera din Piatra Boiului), Szegyestel-Drăcoaia Cave (Sighiştel, Peştera Drăcoaia), Szegyestel-völgyi Caves (peşteri din Valea Sighiştelului) (Kessler 1982), Vársonkolyos-Kis-Magyar Cave (Şuncuiuş, Peştera Napiştileu), Vársonkolyosi Caves (peşteri din Şuncuiuș) (Kessler 1977, Gál 2002) (all in Romania). From sites in Europe outside the Carpathian Basin Q1-2: Spain; Q3: Czech Republic, France, Italy, Malta, Spain; Q4: Austria, Bosnia-Herczegovina, Croatia, France, Germany, Greece, Ireland, Italy, Moldova, Poland, Portugal, Russia, Spain, Switzerland, Ukraine, United Kingdom (Tyrberg 1998).

The genus was reported with extinct species only from Bulgaria (Varshets and Slivnita, Upper Pliocene - Early Pleistocene, MN 17-Q1) as Coccothraustes simeonovi Boev 1998 and C. balcanicus Boev, 1998 (Boev 1998).

- Pyrrhula Linnaeus, 1758

- Pyrrhula † gali Kessler, 2013

Type locality and age: Polgárdi 5, Upper Miocene (MN 13) (Hungary) (Kessler 2013a, 2013b). Corresponds in its characteristics to extant species and genus.

\section{- Pyrrhula $\uparrow$ minor Kessler, 2013}

Type locality and age: Csarnóta 2, Pliocene (MN 15) (Hungary) (Kessler 2013a, 2013b). Corresponds in its characteristics to extant genus but differs in dimensions.

- Pyrrhula pyrrhula Linnaeus, 1758 
Q1: Betfia 9 (Romania) (Gál 2002); Q3: Uppony I/1 (Hungary) (Jánossy 1979); Q4/I: Merkenstein (Austria) (Wettstein \& Mühlhofer 1938); Velika Pecina (Croatia) (V. Malez 1975, 1984, 1988); Budapest-Remetehegyi Niche (Kormos 1914, Lambrecht 1933, Jánossy 1979, 1986), Hámor-Puskaporos Niche (Lambrecht 1912, 1916, 1933, Jánossy 1979, 1986), Hámor-Herman Ottó Cave (Lambrecht 1915, 1933), Pilisszántói I. Niche (Lambrecht 1915, 1933, Jánossy 1979, 1986), Répáshuta-Balla Cave (Lambrecht 1912, 1933) (all in Hungary); Szegyestel-Măgura Cave (Sighiştel, Peştera Măgura) (Romania) (Kessler 1982, 1985, Gál 2002); Q4/II: Legény Cave (Hungary) (Lambrecht 1914); Herkulesfürdő-Rablók Cave (Băile Herculane, Peştera Hoţilor) (Kessler 1981, Gál 2002), Kazánszoros-Climente Cave (Kessler 1981, Gál 2002), Szegyestel-völgyi Caves (Peşteri din Valea Sighiştelului) (Kessler 1982), Szkerisoara-Coiba Mare Cave (Scărişoara, Peştera Coiba Mare) (Kessler 1982, Jurcsák \& Kessler 1986, 1988) (all in Romania). From sites in Europe outside the Carpathian Basin Q3: Croatia, Czech Republic, France, Italy; Q4: Austria, Bulgaria, Croatia, France, Germany, Greece, Ireland, Italy, Poland, Spain, United Kingdom (Tyrberg 1998).

\section{- Pyrrhula sp. foss. indet.}

MN 15: Beremend 26 (Hungary) (Kessler 2010a); based on maxillae and mandibles.

The genus was reported outside the Carpathian Basin from the Late Pliocene - Early Pleistocene, (MN 17 - MQ1) in Varshets - Bulgaria by Boev $(1996,1997)$ and Stránská skála - Czech Republic by Jánossy (1972a).

- Fringilla Linnaeus, 1758

- Fringilla $\uparrow$ kormosi Kessler, 2013

Type locality and age: Polgárdi 5, Upper Miocene (MN 13) (Hungary) (Kessler 2013a, 2013b). It is large size Fringilla species.

\section{- Fringilla $\dagger$ petenyii Kessler, 2013}

Type locality and age: Csarnóta 2, Middle Pliocene (MN 15-16) (Hungary) (Kessler 2013a, 2013b). Its characteristics and dimensions correspond to the extant genus.

\section{- Fringilla montifringilla Linnaeus, 1758}

Q1: Betfia 2 (Kormos 1913, Čapek 1917, Lambrecht 1933, Kessler 1975, Jánossy 1979, Gál 2002), Betfia 9 (Kessler 1975, Gál 2002) (all in Romania); Q4/I: Budapest-Remetehegyi Niche (Hungary) (Kormos 1914, Lambrecht 1933, Jánossy 1979, 1986); Körösmart (Rîpa) (Jánossy in Hamar \& Csák 1969, Kessler 1974a, Gál 2002), Ohábaponor-Bordu Mare Cave (Ohaba Ponor-Peştera Bordu Mare) (Kessler 1985, Jurcsák \& Kessler 1988, Gál 2002, 2003) (all in Romania); Q4/II: Teufelslucke (Austria) (Soergel 1966); Répáshuta-Rejteki Niche (Hungary) (Jánossy 1962, 1979, 1986). From sites in Europe outside the Carpathian Basin Q4: Czech Republic, France, Germany, Italy, Spain, United Kingdom (Tyrberg 1998).

\section{- Fringilla coelebs Linnaeus, 1758}

Q1: Betfia 2 (Kormos 1913, Čapek 1917, Lambrecht 1933, Kessler 1975, Jánossy 1979, Gál 2002), Betfia 9 (Kessler 1975, Gál 2002) (all in Romania); Q2: Kiskóh-Medvék Cave 2 (Chiṣcău, Peștera Urșilor) (Romania) (Kessler 1982, Jurcsák \& Kessler 1988, Gál 2002); Q4/I: Velika Pecina (Croatia) (V. Malez 1975, 1984, 1988); Esküllő-Igric Cave (Aștileu, Peştera Igriţa) (Romania) (Kessler 1985); Q4/II: Kazánszoros-Climente Cave (Kessler1981, Gál 2002), Révi Caves (Peşterile din Vadu Crișului) (Kessler 1982), 
Szkerisoara-Coiba Mare Cave (Scărişoara, Peştera Coiba Mare) (Kessler 1982, Jurcsák \& Kessler 1986, 1988) (all in Romania). From sites in Europe outside the Carpathian Basin Q1-2: Spain, Ukraine; Q3: Croatia, France, Spain; Q4: Austria, Bulgaria, Croatia, Czech Republic, France, Germany, Ireland, Italy, Poland, Spain, Switzerland, Ukraine, United Kingdom (Tyrberg 1998).

\section{- Fringilla sp. foss. indet.}

Localities and age: Litke 2, Lower Miocene (MN 5), Mátraszőlős 2, Middle Miocene (MN 7-8) (Kessler \& Hír 2012b) (all in Hungary).

The genus is known outside of the Carpathian Basin from the Lower Pliocene (MN 16) from Hostalets de Pierola - Spain as Fringilla sp. (Villalta 1963), from the Late Pliocene - Early Pleistocene (MN 17-MQ1) from Varshets - Bulgaria (Boev 1996, 1997); S'Onix (Mallorca) - Spain (Sondaar et al. 1995) and Tarchankut - Ukraine (Vojitsvens'ky 1967) as F. cf. coelebs Linnaeus, 1758.

\section{- Montifringilla Adams, 1858}

\section{- Montifringilla nivalis (Linnaeus, 1766)}

Q4/II: Grosse Offenbergerhöhle (Bocheński \& Tomek 1994); Teufelslucke (Soergel 1966) (all in Austria). From sites in Europe outside the Carpathian Basin Q3: France; Q4: Austria, France, Germany, Italy, Poland, Spain, Ukraine (Tyrberg 1998).

\section{- Loxia Linnaeus, 1758}

\section{- Loxia † csarnotanus Kessler, 2013}

Type locality and age: Csarnóta 2, Pliocene (MN 15) (Hungary) (Kessler 2013a, 2013b). Other locality and age: Beremend 26, Pliocene (MN 15) (Hungary) (Kessler 2013a, 2013b). Its characteristics correspond with the extant genus, but has smaller dimensions.

\section{- Loxia curvirostra Linnaeus, 1758}

Q1: Betfia 9 (Romania) (Gál 2002); Q3/I: Hundsheim (Mlíkovskỳ 2009); Q4/I: Budapest-Remetehegyi Niche (Kormos 1914, Lambrecht 1933, Jánossy 1979, 1986), Hámor-Puskaporos Niche (Lambrecht 1912, 1916, 1933, Jánossy 1979, 1986), Hámor-Herman Ottó Cave (Lambrecht 1915, 1933), Pilisszántói I. Niche (Lambrecht 1915, 1933, Jánossy 1979, 1986), Szilvásvárad-Istállóskői Cave (Lambrecht 1912, 1933, Jánossy 1952, 1955, 1979, 1986) (all in Hungary); Szegyestel-Măgura Cave (Sighiştel, Peştera Măgura) (Romania) (Kessler 1982, 1985, Gál 2002); Q4/II: Herkulesfürdő-Zoltán Cave (Băile Herculane, Peştera Zoltan) (Romania) (Gál 2002). From sites in Europe outside the Carpathian Basin Q3: Czech Republic; Q4: Bulgaria, Croatia, Czech Republic, France, Italy, Poland, Russia, Spain, Ukraine, United Kingdom (Tyrberg 1998).

\section{- Loxia leucoptera J. F. Gmelin, 1789}

From sites in Europe outside the Carpathian Basin Q4: Italy (Tyrberg 1998).

\section{- Loxia pytyopsittacus Borkhausen, 1793}

From sites in Europe outside the Carpathian Basin Q3: France; Q4: Czech Republic, France, Italy (Tyrberg 1998).

\section{- Loxia sp.}

Q4/I: Merkenstein (Wettstein \& Mühlhofer 1938).

From sites in Europe outside the Carpathian Basin Q3: Italy; Q4: Switzerland (Tyrberg 1998). 
The genus was reported as Loxia sp. from Saint-Gerand-le Puy-France (Lower Miocene, MN 2) (Mourer-Chauviré 1995) and as Loxia patevi Boev, 1999 from Varshets - Bulgaria (Upper Pliocene, MN 17) (Boev 1999).

\section{- Fringillidae gen. et sp. indet}

Q4/I: Répáshuta-Balla Cave (Hungary) (Lambrecht 1912, 1933).

The fossil species from this family from Polgárdi 4, 5, Csarnóta 2 and Beremend 26 were innitialy indicated as Fringillidae gen. et sp. indet. by Kessler (2010).

\section{Fam. Emberizidae Vigors, 1831}

\section{- Emberiza Linnaeus, 1758}

\section{- Emberiza † bartkoi Kessler et Hír, 2012}

Type locality and age: Litke 2, Lower Miocene (MN 5) (Hungary) (Kessler \& Hír 2012). In size it resembles a medium-to-large goldfinch.

\section{Emberiza $\uparrow$ pannonica Kessler, 2013}

Type locality and age: Polgárdi 5; Upper Miocene (MN 13) (Kessler 2013a, 2013b). It corresponds to medium-sized extant species (E. citrinella, E. cia, E. cyrlus). The fossil species Emberiza bartkoi Kessler et Hír, 2012 from Litke 2 - Hungary (Lower Miocene, MN 5) seems similar in it sizes to Polgárdi specimen but was described from a distal fragment of a humerus.

\section{- Emberiza $\uparrow$ polgardiensis Kessler, 2013}

Type locality and age: Polgárdi 5, Upper Miocene (MN 13) (Hungary) (Kessler 2013a, 2013b). In its sizes it is similar to the smaller extant species (E. schoeniclus).

\section{- Emberiza † media Kessler, 2013}

Type locality and age: Csarnóta 2, Pliocene (MN 15) (Hungary) (Kessler 2013a, 2013b). It corresponds to characteristics of extant genus.

\section{- Emberiza † parva Kessler, 2013}

Type locality and age: Csarnóta 2, Pliocene (MN 15) (Hungary) (Kessler 2013a, 2013b). Corresponds in its characteristics to extant genus.

\section{- Emberiza † gaspariki Kessler, 2013}

Type locality and age: Beremend 26, Pliocene (MN 15) (Hungary) (Kessler 2013a, 2013b). It corresponds in its characteristics to extant species but has larger dimensions.

\section{- Emberiza cirlus Linnaeus, 1766}

Q4/I: Velika Pecina (V. Malez 1975, 1984, 1988). From sites in Europe outside the Carpathian Basin Q4: Italy, Spain (Tyrberg 1998).

\section{- Emberiza calandra Linnaeus, 1758}

Q1: Betfia 2 (Kormos 1913, Čapek 1917, Lambrecht 1933, Kessler 1975, Jánossy 1979, Gál 2002), Betfia 9 (Kessler 1975, Gál 2002) (all in Romania); Q4/I: Hámor-Puskaporos Cave (Lambrecht 1916, 1933, Jánossy 1979, 1986), Pilisszántói I. Niche (Lambrecht 1915, 1933, Jánossy 1979, 1986) (all in Hungary); Q4/II: Miskolc-Felső forrás (Hungary) (Kessler 2010). From sites in Europe outside the Carpathian Basin the species is unknown.

\section{- Emberiza citrinella Linnaeus, 1758}

Q1: Betfia 9 (Romania) (Gál 2002); Q3: Vindija (Croatia) (M. Malez 1961, V. Malez 1973, 1988, M. Malez \& Rukavina 1979); Uppony I/1 (Hungary) (Jánossy 1979); Q4/I: 
Velika Pecina (Croatia) (V. Malez 1975, 1984, 1988); Varbó-Lambrecht Kálmán Cave (Hungary) (Jánossy 1964, 1979); Q4/II: Teufelslucke (Austria) (Soergel 1966); Herkulesfürdő-Zoltán Cave (Băile Herculane, Peştera Hoţilor) (Romania) (Gál 2002). From sites in Europe outside the Carpathian Basin Q3: Czech Republic, France, Spain; Q4: Austria, Croatia, Czech Republic, France, Germany, Ireland, Italy, Russia, Spain, Ukraine, United Kingdom (Tyrberg 1998).

\section{- Emberiza schoeniclus Linnaeus, 1758}

Q4/I: Merkenstein (Austria) (Wettstein \& Mühlhofer 1938); Hámor-Puskaporos Cave (Hungary) (Lambrecht 1912, 1916, 1933, Jánossy 1979, 1986). From sites in Europe outside the Carpathian Basin Q4: Czech Republic, Italy, Poland, United Kingdom (Tyrberg 1998).

- Emberiza cia Linnaeus, 1766

From sites in Europe outside the Carpathian Basin Q4: Italy, Spain, Ukraine (Tyrberg 1998).

\section{- Emberiza hortulana Linnaeus, 1758}

From sites in Europe outside the Carpathian Basin Q3: France; Q4: France, Italy, Ukraine (Tyrberg 1998).

\section{- Emberiza melanocephala Scopoli, 1769}

From sites in Europe outside the Carpathian Basin Q3: Spain; Q4: Spain (Tyrberg 1998). - Emberiza sp.

Q2: Nagyharsányhegy 1-4 (Hungary) (Jánossy 1979) Q4/I: Novi I, III (Slovakia) (Nehring 1880, Róth 1881, Lambrecht 1912, 1933, Jánossy 1979); Q4/II: Grosse Offenbergerhöhle (Austria) (Bocheński \& Tomek 1994).

The genus is known outside the Carpathian Basin from the Late Pliocene - Early Pleistocene (MN 17-MQ1) sediments from Varshets and Slivnita - Bulgaria (Boev 1996, 1997, 2000) and Stránská skála - Czech Republic (Jánossy 1972).

\section{- Plectrophenax Stejneger, 1882}

- Plectrophenax veterior $\dagger$ Kessler, 2013

Type locality and age: Polgárdi 5, Upper Miocene (MN 13) (Hungary) (Kessler 2013a, 2013b). It corresponds in its characteristics to the extant species.

\section{- Plectrophenax nivalis (Linnaeus, 1758)}

Q4/I: Merkenstein (Austria) (Wettstein \& Mühlhofer 1938); Budapest-Remetehegyi Niche (Kormos \& Lambrecht 1914, Lambrecht 1933, Jánossy 1979, 1986), Cserépfalu-Subalyuk Cave (Jánossy 1979), Hámor-Puskaporos Niche (Lambrecht 1912, 1916, 1933, Jánossy 1979, 1986) (all in Hungary). From sites in Europe outside the Carpathian Basin Q3: France, Ukraine; Q4: Czech Republic, France, Germany, Italy, Spain, Ukraine, United Kingdom (Tyrberg 1998).

- Calcarius Bechstein, 1802

- Calcarius lapponicus (Linnaeus, 1758)

From sites in Europe outside the Carpathian Basin Q4: Austria, United Kingdom (Tyrberg 1998).

- Emberizidae gen et sp. indet.

Q3/I: Betfia 7/4 (Romania) (Kessler 1975); Q4/I: Tatabánya-Kálváriahegy 4. Cave (Hungary) (Gál 2005a, 2005b). 


\section{- Passeriformes fam., gen et sp. foss. indet.}

MN 4: Oberdorf (Austria) (Mlíkovskỳ 1998); MN 5: Litke 2 (Hungary) (Kessler \& Hír 2012); MN 6: Dévényújfalu (Devinska Nova Ves) (Slovakia) (Mlíkovskỳ 2002); Kőalja 2 (Subpiatra) (Romania) (Kessler \& Venczel 2009); MN 7-8: Mátraszőlős 1 (Kessler \& Hír 2012), Mátraszőlős 2 (Gál et al. 2000, Kessler \& Hír 2012), Felsőtárkány (Hír et al. 2001, Kessler \& Hír 2012), Felsőtárkány-Felnémet (Kessler \& Hír 2012) (all in Hungary); MN 13: Polgárdi (Hungary) (Kessler 2010); MN 15: Beremend 26 (Kessler 2010), Csarnóta 2, 4 (Kessler 2010) (all in Hungary); MN 16: Beremend 38 (Kessler 2010), Osztramos 7 (Jánossy 1979) (all in Hungary).

Many bones from this material afterwards were identified to the species level (Kessler \& Venczel 2011, Kessler \& Hír 2012, Kessler 2013a, 2013b).

\section{Conclusions}

The order of songbirds (Passeriformes) is the most numerous one in the avian fauna of the Carpathian Basin, as well as of other areas. This is also the case regarding fossil material, although due to the rudimentary nature of collection methods, as well as difficulties concerning their identification, their numbers only grew in the most extant years. Currently, the remains of 58 genera ( 3 of which are extinct) in 16 families have been identified to the species level $(208+2$ species, of which 118 extinct +2 extinct subspecies). This is supplemented by 23 extinct and 20 extant taxa, which were only identified to the family or genus level.

From the territory of current-day Hungary, new 114 extinct taxa represent Neogene Passeriformes, including the material from Polgárdi (39 taxa), Csarnóta (35 taxa) and Beremend (24 taxa), as well as those described from North Hungary (15 taxa) and Romania (Subpiatra - Köalja) (1 taxon).

- The family Alaudidae is one of the most populated, since it is represented by one extinct genus, 10 extinct and 5 extant species in the fossil material. Extinct ones are known from the Early Miocene up to the Early Pliocene, while extant ones are known from the Quaternary. Their size is between that of sparrows and thrushes, they mostly live in open areas and nest on the ground, hence they relatively often fall prey to predators.

- The family Hirundinidae is also well represented by 6 extinct species and 4 extant ones. Although they are swift flyers, they typically live in groups, so they are also often parts of predators' diets. Extinct species are only from the Late Miocene and the Early Pliocene, while extant species are present in every phase of the Quaternary.

- The family Paridae is represented by small sedentary insectivore species. 5 extinct and 7 extant species were identified from the fossil materials. Similar to the Hirundinidae, extinct representatives are only found in the Late Miocene and Early Pliocene, while extant ones are from the Quaternary. Although this phenomenon appears in the case of numerous other families as well, its causes are unclear. It can be due to improper taphonomic conditions, the relatively low number of fossil material, or the improper geological classification of the sites.

- Sittidae, Certhidae and Tichodromidae are families with similar ways of life and sizes, with few species. While the former two families are sedentary insectivores living on barks 
of woodland trees, the sole representative of the third family is migratory and lives on the ledges of cliffs, and differs with its pompous colors from its brown relatives. Even though they are only represented by 4 species in the current fauna, and only two of those are present in the fossil material in the Early and Late Pleistocene and Holocene, 6 extinct species are known from the Late Miocene and Early Pliocene, as well as two pieces of not fully identified material from the Middle Miocene. Their remains also prove the presence of their habitats (woodlands, as well as bare ledges of cliffs) in these time periods

- The family Muscicapidae is one of the richest regarding the number of species. Apart from the 18 extinct species, the remains of 11 extant species were identified from the time period between the Early Miocene and Early Pliocene, as well as from the Quaternary. Of them, nightingales and flycatchers are represented by one extinct species each in every era. They are sparrow-sized, grey or brown migratory insectivores living among bushes or trees. Their presence proves these former conditions.

- The Turdidae family is quite well represented within the fossil material with one extinct genus, 9 extinct species and 6 extant species. The existence of the extinct and much-debated genus (Turdicus) was proven from materials from Lower, Middle, and Upper Miocene materials. Such is also the case with the extinct species (Turdoides borealis) of the northern genus. The extant Turdus genus is represented by 5 extinct species in the Late Miocene and Early Pliocene material, while extant species are more numerous; they are sedentary or migrate, due to their sizes and high numbers they often fall prey to predators.

- The Sylviidae family is another populated one. Apart from 16 extinct species (as well as several finds from the Miocene that had not been fully identified), it is only represented by 4 extant species within the fossil material. The extinct species, as with the families discussed so far, were classified from the Late Miocene and Early Pliocene. Whitethroats, warblers, grasshopper warblers, leaf warblers, icterine warblers and kinglets have gray-brown feathers, they are insectivores and migrate. They live among bushes and trees, thus, their presence indicates this kind of habitat.

- The Motacillidae family (wagtails and pipits), however, consists of birds, about the size of sparrows or larger, living in open areas. They are insectivores and mostly migratory. Apart from 5 extinct species and a few not fully identified finds, they are represented by 8 extant species. Extinct ones were classified from the Middle Miocene to the Early Pleistocene material, thus, representatives of the family from the Middle Miocene to extant times are continuously present in the Carpathian Basin.

- Oriolidae, Bombycillidae, Cinclidae and Troglodytidae are all typically single-species families. Despite this, they are also present within the fossil material, both with their extinct and extant species. Bombycillidae and Cinclidae have three extinct species each, while Oriolidae and Troglodytidae have one each. What is more, the former ones were present from the Early Miocene to the Early Pliocene, while the latter ones only from the Early Pliocene and the Late Miocene. Extant species are known from almost the whole timeframe of the Quaternary. Considering appearance, size and way of life, however, they are quite different families. While the Golden Oriole is a species of relatively larger size (similar to larger blackbirds) with colorful feathers, it lives in woodlands, it is insectivore and migratory. The bohemian waxwings are seed-eating, migrating birds of the taiga with sizes of smaller 
blackbirds. Wrens are rather small birds with brownish feathers, living and nesting on the ground of bushy areas; they feed on insects and are sedentary. The Dipper is a species living around creeks, looking for prey in their beds. Their size is similar to blackbirds, and they are sedentary. Their presence proves that these habitats were present in the total timespan of the Neogene and the Quaternary inside the Carpathian Basin.

- Prunellidae are sparrow-sized birds living among woodlands and bushes with graybrown feathers. They are represented by a few extinct as well as extant species within the fossil material. While the extinct species are birds of the Late Miocene and Early Pliocene, the extant ones are only known from the Late Pleistocene and the Holocene.

- Members of the Laniidae family typically live in bushy/open areas. Their size is between that of sparrows and thrushes, their diet consists of invertebrates, and they are migratory. They are represented by 5 extinct and 4 extant species. The extinct ones lived in the timeframe of the Middle Miocene and Early Pliocene, while extant ones in the whole of the Quaternary. Their not fully identified extinct types are known from the Middle Miocene, as well as the Early and Late Pliocene.

- Extant forms of the Sturnidae family represent two completely different types that are present in the Quaternary, with one species each. The Common Starling lives in the woods and in open areas and reed beds, roams in large flocks, and feeds on insects and fruit, while the Rosy Starling is a migrating insectivore that lives in open areas. The former is present in the whole of the Quaternary, while the latter is only known from the Late Pleistocene. The 4 extinct species were defined and described with different sizes from the Late Miocene and the Early Pliocene.

- The Passeridae and Fringillidae families are closely related, and have many similarities as well. The latter is also rich in species. Three extinct species are known of the former family from the Late Miocene and Early Pliocene (apart from two extant species identified from the Quaternary). The latter family, however, is represented by 11 extinct species from the Late Miocene and Early Pliocene as well, but also by several not fully identified taxa from the Early and Middle Miocene and the Late Pliocene. The remains of 12 extant species were identified from the Quaternary. Their sizes vary between sparrows and smaller blackbirds, they are usually colorful seed-eating sedentary species. In nesting periods, they live in woodland area, in these times they also eat insects (with the exception of Loxia). They are quite frequent within the fossil material as well.

- The Emberizidae family consists of species living in more open areas, feeding on insects and seeds, and are mostly sedentary. Their sizes range from sparrows to starlings. They are represented by 6 extinct and 5 extant species. The former are known from the Late Miocene and Early Pliocene, while the latter are known from the Quaternary, from several sites.

\section{Acknowledgements}

The author wishes to express his deep gratitude to referees for their help in the revision of the text and to József Vuts and Lóránd Abos for the language revision. 


\section{References}

Bocheński, Z. M. \& Tomek, T. 1994. Fossil and subfossil bird remains from five Austrian caves. - Acta Zoologica Cracoviensia 37: 347-358.

Boev, Z. N. 1996. Tertiary avian localities of Bulgaria. - In: Mlí́kovský, J. (ed.) Tertiary avian localities of Europe. - Acta Universitatis Carolinae, Geologica 39: 541-545.

Boev, Z. N. 1997. Chauvireria balcanica gen. n., sp. n. (Phasianidae - Galliformes) from the Middle Villafranchian of Western Bulgaria. - Geologica Balcanica 27: 69-78.

Boev, Z. N. 1998. Late Pliocene hawfinches (Coccothraustes Brisson, 1760) (Aves: Fringillidae) from Bulgaria. - Historia Naturalis Bulgarica 9: 87-99.

Boev, Z. N. 1999. Earliest finds of crossbills (genus Loxia) (Aves: Fringillidae) from Varshets (NW Bulgaria). - Geologica Balcanica 29(3-4): 51-57.

Boev, Z. N. 2000. Neogene avifaunas of Bulgaria. - Vertebrata Palasiatica 38 (Suppl.): 2-10.

Boev, Z. N. 2012. Neogene Larks (Aves: Alaudidae (Vigors, 1825) from Bulgaria. - Acta Zoologica Bulgarica 64(3): 295-318.

Brodkorb, P. 1960. How many species of birds have existed? - Bulletin of the Florida State Museum, Biological Sciences 5: 41-53.

Čapek, V. 1917. A püspökfürdői preglaciális madárfauna [The Preglaciale bird fauna from Püspökfürdő]. Barlangkutatás 5: 66-74. (in Hungarian)

Clot, A., Chaline, J., Heintz, E., Jammot, D., Mourer-Chauviré, C. \& Rage. J. C. 1976. Montoussé 5 (HautesPyrénées), un nouveau remplissage de fissure à faune de vertébrés du Pléstocène inférieur [Montoussé 5 (Hautes-Pyrénées), a new fissure filling with vertebrate fauna of the Lower Pleistocene]. - Géobios 9: 511514. (in French)

Döppes, D. \& Rabeder, G. (eds.) 1997. Pliozäne und Pleistozäne Faunen Österreichs. - Mitteilungen der Kommission für Quartärforschung der Österreichischen Akademie der Wissenschaften [Pliocene and Pleistocene Fauns of Austria - Communications from the Commission for Quaternary Research of the Austrian Academy of Sciences]. Vol. 10. - Wien (in German)

Fischer, K. \& Stephan, B. 1977. Vogelknochenfunde aus Quartären Ablagerungen Südwestrumaniens [Bird bones from Quaternary deposits in Southwest Romania]. - Annalen für Ornithologie 1: 79-90. (in German)

Fladerer, F. A. 1993. Neue Daten aus jung- und mittelpleistozänen Höhlensedimenten im Raum Peggau-Deutschfeistritz, Steiermark [New data from young and Middle Pleistocene cave sediments in the area Peggau-Deutschfeistritz, Styria]. - Fundberichte aus Österreich 31: 369-374. (in German)

Freudenthal, M. \& Kordos, L. 1989. Cricetus polgardiensis sp. nov. and Cricetus kormosi Schaub, 1930 from the Late Micene Polgárdi localities (Hungary). - Scripta Geologica 89: 71-95.

Gál, E. 1998. Avifauna fosilă a peșterii Gura Cheii de la Râșnov (Jud Brașov) [Fossil bird fauna of the Gura Cheii Cave from Râșnov (Brașov County)]. - Studia Universitatis Babeș-Bolyai, Biologia 43(1-2): 8893. (in Romanian)

Gál, E. 2002. Avifauna pleistocena a Romaniei [Notpublished doctoral thesis: Pleistocene birds faunas from Romania]. - Bucuresti (in Romanian)

Gál, E. 2003. Bird remains from two Romanian caves: Curata Cave (Nandru) and Bordu Mare Cave (Ohaba Ponor). - ICAZ 2002, Abstracts, 56.

Gál, E. 2004. The Neolitic avifauna of Hungary within the context of the Carpathian Basin. - Antaeus 27: $273-286$.

Gál, E. 2005a New evidence of fowling and poultry keeping in Pannonia, Dacia and Moesia during the period of the Roman Empire. - In: Gruppe, G. \& Peters, J. (eds.) Feathers, grit and symbolism. Birds and humans in the Ancient Old and New Worlds. - Proceedings of the $5^{\text {th }}$ Meeting of the ICAZ Bord Working Group, Munich, Germany, 26-30 July 2004. - Documenta Archaeobiologiae 3. Verlag Marie Leidorf GmbH.Rahden/Westf., pp. 301-316.

Gál, E. 2005b Animal remains from archaeological excavations in North-Eastern Hungary. - In: Gál, E., Juhász, I. \& Sümegi, P. (eds.) Environmental Archaeology in North-Eastern Hungary. - Varia Archaeologica Hungarica 19: 139-174.

Gál, E. 2007. Fowling in lowlands. Neolithic and Chalcolithic bird exploitation in South-East-Romania and the Great Hungarian Plain. - Archaeolinqua, Series Minor 24., Budapest

Gál, E., Hír, J., Kessler, E., Kókay, J., Mészáros, L. \& Venczel, M. 1998-1999. Középső-miocén ősmaradványok, a Mátraszőlős, Rákóczi-kápolna alatti útbevágásból. I. A Mátraszőlős 1. lelőhely [Middle Miocene fossils from the sections at the Rákóczi Chapell at Mátraszőlős. I. Mátraszőlős 1. site]. - Folia Historico Naturalia Musei Matraensis 23: 33-78. (in Hungarian with English Summary) 
Gál, E., Hír, J., Kessler, E., Kókay, J. \& Vencel, M. 2000. Középső-miocén ősmaradványok a Mátraszőlős, Rákóczi-kápolna alatti útbevágásból. II. A Mátraszőlős 2. lelőhely [Middle Miocene fossils from the sections at the Rákóczi Chapell at Mátraszőlős. I. Mátraszőlős 2. site]. - Folia Historico Naturalia Musei Matraensis 23: 33-78. (in Hungarian with English Summary)

Gál, E. \& Kessler, E. 2006. Songbird remains from the Miocene (Middle Sarmatian) site Credinta (Dobrogea, South-East Romania). - In: Csiki, Z. (ed.) Volume dedicated to Dan Grigorescu on his $65^{\text {th }}$ birthday. - University of Bucharest Printing House

Harrison, C. J. O. \& Stewart, J. R. 1999. Avifauna. - In: Roberts, M. B. \& Parffit, S. A. (eds.) A Middle Pleistocene hominid site at Eartham Quarry, Boxgrove, West Sussex. - English Heritage Archaeological Report (London) 17: 187-196.

Harrison, C. J. O. 1979. Small non-passerine birds of the Lower Tertiary as exploiters of ecological niches now occupied by passerines. - Nature 281: 562-563.

Hír, J., Kókay, J., Venczel, M., Gál, E. \& Kessler, E. 2001. Előzetes beszámoló a felsőtárkányi „Gödör-kert” öslénytani lelőhelykomplex újravizsgálatáról [A preliminary report on the revised investigation of the paleontological locality complex “Gödör-kert” at Felsőtárkány, Northern Hungary]. - Folia Historico-Naturalia Musei Matraensis 25: 41-65. (in Hungarian with English Summary)

Horáček, I. 1985. Survey of the fossil vertebrate localities Včeláre 1-7. - Časopis pro Mineralogii a Geologii 30: 353-366.

Jánossy, D. 1952. Az Istállóskői barlang aurignaci faunája [The Aurignacian cave fauna from Istállóskő]. Földtani Közlöny 82(4-6): 181-203. (in Hungarian)

Jánossy, D. 1954. Fossile Ornis aus der Höhle von Istállóskő [Fossile birds from the Istállóskő Cave]. - Aquila 55-58: 205-223. (in German)

Jánossy, D. 1955. Die Vogel-und Saugetierreste der Spätpleistozänen Schichten der Höhle von Istállóskő [The bird and mammalian remains of the Late Pleistocene Layers of the Istállóskő Cave]. - Acta Archaeologica Academiae Sciencentiarum Hungaricae 5: 149-181. (in German)

Jánossy, D. 1962. Eine fossile Vogelfauna aus den Mosuterien-Schichten der Subalyuk-Höhle im Bükk-Gebirge [A fossil avifauna from the Mousterian layers of Subalyuk cave in the Bükk Mountains]. - Aquila 67-68., 175-188. (in German)

Jánossy, D. 1965. Vogelreste aus den altpleistozänen Ablagerungen von Voigtstedt in Thüringen [Bird remains from the old Pleistocene deposits of Voigtstedt in Thuringia]. - Paläontologische Abhandlungen (A) 2: 336-361. (in German)

Jánossy, D. 1972. Die mittelpleistozäne Vogelfauna der Stránská Skála [The Middle Pleistocene bird fauna of Stranská Skálá]. - Anthropos 21(12): 35-64. (in German)

Jánossy, D. 1974a Die mittelpleistozäne Vogelfauna von Hundsheim (Niederösterreich) [The Middle Pleistocene avifauna of Hundsheim (Lower Austria)]. - Sitzungsberichte der Österreichischen Akademie der Wissenschaften, Mathematisch-Naturwissenschftliche Klasse, Abteilung I. 182: 211-257. (in German)

Jánossy, D. 1974b Upper Pliocene and Lower Pleistocene bird remains from Poland. - Acta Zoologica Cracoviensia 19: 531-564.

Jánossy, D. 1978. Új finomrétegtani szint Magyarország pleisztocén őslénytani sorozatában [A new fine stratigrafic level in Paleontological series at Hungarian Pleistocene]. - Földrajzi Közlemények 26(1-3): 161174. (in Hungarian)

Jánossy, D. 1979. Plio-pleistocene Bird Remains from the The Carpathian Basin. IV. Anseriformes, Gruiformes, Charadriiformes, Passeriformes. - Aquila 85: 11-39.

Jánossy, D. 1980. Plio-pleistocene Bird Remains from the The Carpathian Basin. VI. Systematical and Geographical Catalogue. - Aquila 87: 9-22.

Jánossy, D. 1981. Die altpleistozänen Vogelfaunen von Deutsch-Altenburg 2 und 4 (Niederösterreich) [The Early Pleistocene bird fauna of Deutsch-Altenburg 2 and 4 (Lower Austria)]. - Beiträge zur Palaontologie von Österreich 8: 375-391. (in German)

Jánossy, D. 1982. Új adatok az európai madárfauna kialakulásáról [New data on the evolution of European bird fauna]. - Földtani Közlemények 112: 449-453. (in Hungarian)

Jánossy, D. 1983. Die mittelpleistozäne Vogelfauna von Přezletice bei Prag (ČSSR) [The Pleistocene bird fauna of Přezletice near Prague (CSSR)]. - In: Heinrich, W-D. (ed.) Wirbeltier-Evolution und Faunenwandel im Känozoikum [Vertebrate Evolution and Faunal Change in the Cenozoic]. - Schriftenreihe für Geologische Wissenschaften 19-20: 247-269. (in German)

Jánossy, D. 1986. Pleistocene vertebrate faunas of Hungary. - Budapest, Akadémiai Kiadó \& Amsterdam, Elsevier 
Jánossy, D. 1991. Late Miocene bird remains from Polgárdi (W-Hungary). - Aquila 98: 13-35.

Jánossy, D. 1992. Lower Pleistocene bird remains from Beremend (S-Hungary, Loc. 15. and 16.). - Aquila 99: 9-25.

Jánossy, D. 1993. Bird remains from the Upper Miocene (MN 9) of Rudabánya (N-Hungary). - Aquila 100: 53-70.

Jánossy, D. 1995. A Late Miocene avifauna from Polgárdi, western Hungary. - In: Peters, D. S. (ed.) Acta Palaeornithologica. - Courier Forschunsinstitut Senckenberg 181: 203-206.

Jánossy, D. 1996. Lower Pleistocene vertebrate faunas from the localities 16 and 17 of Beremend (Southern Hungary). - Fragmenta Mineralogica et Paleontologica 18: 91-102.

Jánossy, D. \& Kordos, L. 1976. Az Osztramos gerinces lelőhelyeinek faunisztikai és karszt-morfológiai áttekintése (1974-ig) [The faunistical and karst-morphological review of paleontological localities for Vertebrates at Osztramos (to 1974)]. - Fragmenta Mineralogica et Palaeontologica 8: 39-72. (in Hungarian and English)

Jurcsák, T. \& Kessler, E. 1986. Evoluţia avifaunei pe teritoriul României (I) [Evolution of avifauna on Romanian territory]. - Crisia 16: 577-615. (in Romanian with English Summary)

Jurcsák, T. \& Kessler, E. 1988. Evoluţia avifaunei pe teritoriul României (III) [Evolution of the avifauna in the territory of Romanian, Part III.]. - Crisia 18: 647-688. (in Romanian with English Summary)

Kessler, E. 1974a Date noi asupra avifaunei fosile a Dealului Burzău - Râpa [New data to fossil bird fauna from Burzau Hill (Village Rîpa)]. - Nymphaea 2: 159-167. (in Romanian)

Kessler, E. 1974b Contribuții noi la avifauna depozitelor hidrotermale postglaciare de la Băile 1 Mai - Oradea, jud. Bihor [New contributions to postglaciale avifauna and hydrothermal sediments from Băile 1 Mai Oradea, Bihor County]. - Tibiscus 11: 123-128. (in Romanian)

Kessler, E. 1975. Contribuţii noi la studiul avifaunei fosile de la Betfia, jud. Bihor [New contributions to fossile avifauna from Betfia, Bihor County]. - Nymphaea 3: 53-59. (in Romanian)

Kessler, E. 1977. Avifauna postglaciară de la Vadu Crişului, jud. Bihor [Postglaciar avifauna from Vadu Crişului, Bihor County]. - Tibiscus (1977): 255-260.

Kessler, E. 1981. Noi date privind avifauna pleistocenă a Văii Ierului (jud. Bihor) [New data on the Pleistocene bird fauna of Valea Ierului (Bihor County)]. - Nymphaea 8-9: 259-264. (in Romanian)

Kessler, E. 1982. Avifauna fosilă şi subfosilă a Munţilor Apuseni [Fossil and subfossil bird fauna from Apuseni Mountains]. - Nymphaea 10: 171- 181. (in Romanian)

Kessler, E. 1985b Contribuţii noi la studiul avifaunelor cuaternare din România [New contributions to the study of Quaternary bird faunas in Romania]. - Crişia 15: 485-491. (in Romanian)

Kessler, J. 2010. Új eredmények a Kárpát-medence neogén és negyedidőszaki madárvilágához, III. rész [New results with regard to the Neogene and Quaternary Avifauna of the Carpathian basin. Part III.]. - Földtani Közlöny. 140(1): 53-72. (in Hungarian)

Kessler, J. \& Hír, J. 2012. Észak-Magyarország madárvilága a miocénben II. [The avifauna in North Hungary during the Miocene, Part II.]. - Földtani Közlöny 141(2): 149-168. (in Hungarian with English Summary)

Kessler, E. 2013a Neogene songbirds from Hungary (Aves, Passeriformes) faunae from Hungary. - Hantkeniana 2013: 37-149.

Kessler, J. 2013b A Kárpát-medence madárvilágának őslénytani kézikönyve [Palaeornithological Handbook of birds fauna from Carpathian Basin]. - Könyvműhely Kiadó Miskolc (in Hungarian)

Kessler, E. \& Gál, E. 1998. Resturi fosile si subfosile de pasari in siturile paleolitice si neolitice din Cheile Turzii si Cheile Turenilor (Judetul Cluj) [Fossil and sub-fossil remains of birds in the Paleolithic and Neolithic sites of the Turzii Gorge and the Gorges of Tureni (Cluj County)]. - Angvistia 3: 9-122. (in Romanian)

Kessler, J. \& Hír, E. 2012. Észak-Magyarország madárvilága a miocénben II. rész [The avifauna in North Hungary during the Miocene. Part II.]. - Földtani Közlöny 142(2): 149-168. (in Hungarian)

Kessler, E. \& Venczel, M. 2009. Bird remains from the Middle Miocene of Subpiatră (W-Romania). - Nymphaea, Folia naturae Bihariae 36: 27-36.

Kessler, E. \& Venczel, M. 2011. A new passeriform bird from the Middle Miocene of Subpiatră (W-Romania). - Nymphaea, Oradea, 38: 17-22.

Kordos, L. 1984. A bodajki Rigó-lyuk újholocén kitöltésének vizsgálata [Investigating the Holocene filling of the Bodajk-Rigó Hole]. - Folia Musei Historico-Naturalis Bakonyensis 3: 31-42. (in Hungarian)

Kormos, T. 1911. Die pliozäne Knochenfund bei Polgárdi (vorläufiger Bericht) [The pliocene bone find at Polgárdi (preliminary report)]. - Földtani Közlöny 41: 171-189. (in German) 
Kormos, T. 1913. Kleinere Mitteilungen aus dem ungarischen Pleistozän [Small releases from the Hungarian Pleistocene]. - Centralblatt für Mineralogie, Geologie und Palaontologie 1913: 13-17. (in German)

Kormos, T. 1914. Az 1913. évben végzett ásatásaim eredményei [The results of the excavations carried in the year 1913]. - A Magyar Királyi Földtani Intézet 1913. évi jelentése, pp. 498-505., 531-540. (in Hungarian)

Kretzoi, M. 1952. Die Raubtiere der Hipparion-fauna von Polgárdi [The predators of the Hipparion fauna of Polgárdi]. - Jahrbuch Ungarische Geological Anstalt 40: 1-12. (in German)

Kretzoi, M. 1956. A Villányi hegység alsó pleisztocén gerinces faunái [Ostrich remains from Kisláng, Fejér County]. - Geologica Hungarica 27: 1-264. (in Hungarian)

Kretzoi, M. 1962. A csarnótai fauna és faunaszint [The fauna and fauna level of the Csarnóta]. - A Magyar Állami Földtani Intézet Évi Jelentése az 1959. évröl, pp. 297-395. (in Hungarian)

Kretzoi, M. 1969. A magyarországi quarter és pliocén szárazföldi biosztratigráfiájának vázlata [Outline of terrestrial biostratigraphy of the Hungarian Quaternary and Pliocene]. - Földrajzi Közlemények 18(3): 179204. (in Hungarian)

Kretzoi, M. 1975. Fontosabb szórványleletek a MÁFI gerinces gyüjteményében (2 studies) [The most important Vertebrate finds of the Hungarian Geological Institute]. - A Magyar Állami Földtani Intézet Évi jelentése 1975 évröl, pp. 401-415. (in Hungarian)

Kretzoi, M. 1977. The fauna of small vertebrates of the Middle Pleistocene at Petralona. - Anthropos (Athena) 4: 131-143.

Lambrecht, K. 1912. Magyarország fossilis madarai - Die fossilen Vögel Ungarns [The Fossil birds of Hungary]. - Aquila 19: 288-320. (in Hungarian and German)

Lambrecht, K. 1913. Neueren Daten zur fossilen Ornis unseren Höhlen [Extant data for fossil birds in our caves]. - Barlangkutatás 1: 173. (in German)

Lambrecht, K. 1915. Fossilis nagy fülesbagoly (Bubo maximus Flemm.) és egyéb madármaradványok a magyarországi pleisztocénből [Fossil's Big Eared Owl (Bubo maximus Flemm.) and other bird remains in Hungarian Pleistocene]. - Aquila 22: 177-187. (in Hungarian)

Lambrecht, K. 1916. Az első magyar preglaciális madárfauna. Die erste ungarische praglaziale Vogelfauna [The first Hungarian Preglaciale bird fauna]. - Aquila 22: 165-172. (in Hungarian and German)

Lambrecht, K. 1933. Handbuch der Palaornithologie [Handbook of Palaeornithology]. - Gebrüder Borntraeger, Berlin (in German)

Malez, M. 1961. Pecinska hijena iz indije kod Voce [The Cave Hyaenas come from Vindija Cave]. - Geologiceskij Vjesnik 14: 221-244. (in Croatian)

Malez, V. 1973. Fosilne ptice Jugoslavije [Fossil birds from Yugoslavia]. - Unpublished Dissertation, Zagreb University, pp. 1-97. (in Croatian)

Malez, M. 1975. Ornitofauna iz kvartarnih naslaga velike pecine na ravnoj gori u sjeve-rozapadnoj Hrvatskoj [Ornithofauna from the Quaternary sediments of a large cave on a flat mountain in Northwestern Croatia]. - Larus 26-28: 45-54. (in Croatian)

Malez, M. \& Rukavina, D. 1975. Kristurbacijske pojave u gornjopleistocenskim nasla-gama pecine Vindije kod Donje Voce u sjeverozapadnoj Hrvatskoj [Cristurbation appearance in Upper Pleistocene at Vindija cave in Northwestern Croatia]. - Rad Hazu knj. 371: 245-265. (in Croatian)

Malez, V. 1973. Fosilne ptice Jugoslavije [Fossil birds from Yugoslavia]. - Unpublished Dissertation, Zagreb University, pp. 1-97. (in Croatian)

Malez, V. 1984. Paleornitološki ostaci iz kvartarnih naslaga nekih spilja Hrvatske i Slovenije [Paleornithological remains from the Quaternary Sediments of some Croatian and Slovenian caves]. - In: Deveti jugoslavenski speleoloski kongres, Zbornik predavanja, pp. 711-719. (in Croatian)

Malez, V. 1988. Pleistocenska ornitofauna iz spilje Vindije u sjevernozapadnoj Hrvatskoj [Pleistocene bird fauna from Vindija cave in Northwestern Croatia]. - Rad Jugoslavenske Akademije Znanosti i Umjetnosti, Varazdin 2: 31-203. (in Croatian)

Malez-Bačic, V. 1979. Pleistocenska ornitofauna iz Šandalje u Istri te njezino stratigrafsko i paleoekološko značenje [Pleistocene bird fauna from Šandalja in Istria and its stratigraphic and paleoecological significance]. - Palaeontologia Jugoslavica 21: 1-46. (in Croatian)

Milne-Edwards, A. 1869-1871. Recherches anatomiques et paléontologiques pour servir àl'histoire des oiseaux fossiles de la France, Vol. 2. [Anatomical and paleontological research to serve the history of fossil birds of France, Vol. 2.]. - Paris, G. Masson (in French)

Mlíkovský, J. 1989. Ptáci staršího a středního pleistocénu Československa: současný stav aperspektivy výzkumu [Birds of the Early and Middle Pleistocene of Czechoslovakia: The Current State of Research 
Approach]. - In: Seitl, L. (ed.) Současný stav a perspektivy výzkumu kvartéru v ČSSR [The Current State and Perspectives of the Quaternary Research in Czechoslovakia]. - Brno: Moravské muzeum \& Universita J. E. Purkyně, pp. 63-67. (in Czech)

Mlíkovský, J. 1995. Early Pleistocene birds of Stránská skála: 1. Musil's talus cone. - In: Musil, R. (ed.) Stránská skála Hill: Excavations of open-air sediments 1964-1972. - Anthropos (Brno) 26: 111-126. (in Czech)

Mlíkovský, J. (ed.) 1996. Tertiary avian localities of Europe. - Acta Universitatis Carolinae, Geologica 39: 517-848.

Mlíkovský, J. 1998. Early Pleistocene birds of Deutsch-Altenburg, Austria. - Acta Societatis Zoologicae Bohemicae 62: 135-141.

Mlíkovský, J. 2002. Cenozoic Birds of the World. Part 1: Europe. - Ninox Press, Praha

Mlíkovský, J. 2009. Middle Pleistocene birds of Hundsheim, Austria. - Journal of the National Museum (Prague), Natural History Series 177(7): 69-82.

Montoya, P. 1999. La fauna del Pleistoceno inferior de la Sierra de Quibas (Abanilla, Murcia) [The fauna of the Lower Pleistocene of the Sierra de Quibas (Abanilla, Murcia)]. - Estudios Geológicos 55 (3-4): $127-$ 161. (in Spanish)

Montoya, P. 2001. Une faune très diversifiée du Pléistocène inférieur de la Sierra de Quibas (province de Murcia, Espagne) [A very diverse fauna from the Lower Pleistocene of the Sierra de Quibas (province of Murcia, Spain)]. - Comptes Rendus de l'Académie des Sciences (Paris), Sciences de la Terre et des Planètes 332: 387-393. (in French)

Mourer-Chauviré, C. 1975. Les oiseaux du Pléistocène moyen et supérieur de France [The Middle and Upper Pleistocene birds of France]. - Documents des Laboratoires de Géologie de la Faculté des Sciences de Lyon 64: 1-624. (in French)

Mourer-Chauviré, C. 1995. Dynamics of the avifauna during the Paleogene and the Early Neogene of France. Settling of the extant fauna. - Acta Zoologica Cracoviensia 38(3): 325-342.

Mourer-Chauviré, C., Hugueney, M. \& Jonet, P. 1989. Découverte de Passeriformes dans l'Oligocène supérieur de France [Discovery of Passeriformes in the Upper Oligocene of France]. - Comptes Rendus Hebdomadaires de l'Académie des Sciences (Paris), Sciences de la Terre et des Planètes 309: 843-849. (in French)

Nagy, J. 2020. Biologia Futura: rapid diversification and behavioural adaptation of birds in response to Oligocene-Miocene climatic conditions. - Biologia Futura 71: 109-121.

Nehring, A. 1880. Uebersicht über vierundzwanzig mitteleuropäische Quartär-Faunen [Survey of twenty-four Central European Quaternary fauna]. - Zeitschrift der Deutschen Geologischen Gesellschaft 32: 468-509. (in German)

Noriega, J. I. \& Chiappe, L. 1993. An Early Miocene Passeriform from Argentina. - The Auk 110(4): 936-938.

Pike-Tay, A., Bartosiewicz, L., Gál, E. \& Whitle, A. 2004. Body part representation and seasonility: sheep/ goat, bird and fish remains from Early Neolithic Ecsegfalva 23 SE Hungary. - Journal of Taphonomy 2(4): 221-246.

Pongrácz, L. 1999. A beremendi Szőlő-hegy természettudományi kutatásának 150 éve [150 years of natural science research on Beremend Szölö-Hill]. - Petényi emlékkönyv, Beremend

Portis, A. 1887. Contribuzioni alla ornitolitologia italiana. Parte II. [Contributions to Italian ornitholithology. Part II.]. - Memorie Regia Accademia Scienze (Torino) (2) 38: 181-203. (in Italian)

Róth, S. 1881. Szepesmegye néhány barlangjának leirása [Description of some caves in Szepes County]. Mathematikai és Természettudományi Közlemények 16: 613-648. (in Hungarian)

Soergel, E. 1966. Die Vogelreste [The Bird Remains]. - In: Ehrenberg, K. (ed.) Die Teufels-oder Fuck-senlucke bei Eggenburg (NÖ) [The Devil or Fuck senlucke at Eggenburg (NA)]. - Denkschriften der Mathematisch-Naturwissenschftliche Klasse der Österreichischen Akademie der Wissenschaften, pp. 93-107. (in German)

Sondaar, P. Y., Mcminn, M., Segui, B. \& Alcover, J. A. 1995. Interès paleontològic del jaciments càrstic de les Gimnésies i les Pitiüses [Paleontological interest of the karstic deposits of the Gymnasiums and the Pitiusas]. - Endins 20: 155-170. (in Spanish)

Tassi, M. 2006. Szendrő-Felsővár állatcsontanyaga [Animal bone material form Szendrő- Felsővár]. - Visegrádi Múzeumok Közleményei: 15-30. (in Hungarian)

Tchernov, E. 1980. The Pleistocene Birds of Ubeidiya, Jordan Valley. - In: The Pleistocene of the Central Jordan Valley. The excavation at Ubeidiya. - The Israel Academy of Sciences Humanities, Jerusalem, pp. 1-83.

Tyrberg, T. 1998. Pleistocene birds of the Palearctic: a catalogue. - Cambridge, Mass.: Nuttall Ornithological Club 
Villalta, J. F. de 1963. Las aves fósiles del Mioceno español [Fossil birds of the Spanish Miocene]. - Boletín de la Real Sociedad Española de Historia Natural Geología 61: 263-285. (in Spanish)

Vojinstvens'kyj, M. A. 1967. Iskopaemaja ornitofauna Ukrainy [The fossil avifauna of Crimea]. - Prirodnaja Obstanovka i Fauni Prollogo 3: 3-76. (in Russian)

Wettstein, O. V. \& Mühlhofer, F. 1938. Die Fauna der Höhle von Merkenstein in N-Ö [The fauna of the Merkenstein cave in North Austria]. - Archiv Naturgeschichte, New Series 7(4): 514-558. (in German)

Zelenkov, N. 2011. Neogene Birds of Central Asia. - PhD Thesis, Paleontological Institute, RAN, Moscow

Zelenkov, V. N. \& Kurochkin, E. N. 2012. The first representative Pliocene assemblages of passerine birds in Asia (Northern Mongolia and Russian Transbaikalia). - Geobios 45(3): 323-334. DOI: 10.1016/j.geobios.2011.10.001

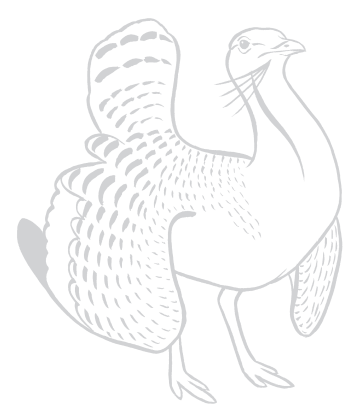

